\title{
An assessment of health risks posed by consumption of pesticide residues in fruits and vegetables among residents in the Kampala Metropolitan Area in Uganda
}

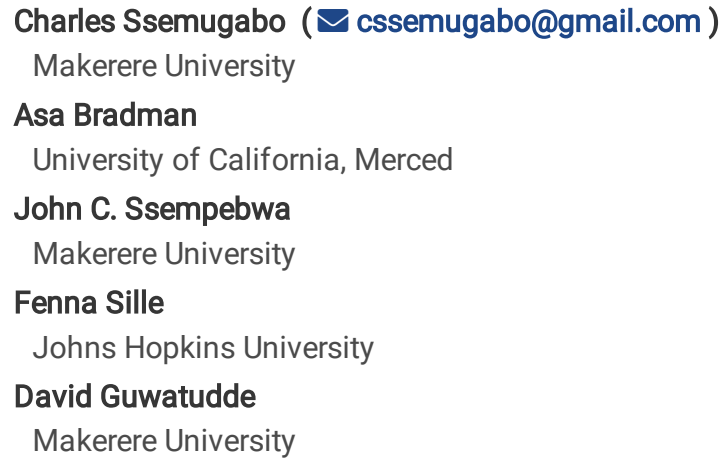

\section{Research Article}

Keywords: Maximum residual limits, Hazard quotient, Estimated daily intake, Acceptable daily intake, Uganda.

Posted Date: February 28th, 2022

DOI: https://doi.org/10.21203/rs.3.rs-1338563/v2

License: (c) (i) This work is licensed under a Creative Commons Attribution 4.0 International License. Read Full License 


\section{Abstract \\ Background}

Pesticide use for fruits and vegetable production in Uganda may result in presence of residues on produce which may pose health risks to consumers. Uganda does not have an established system for monitoring pesticide residues in fruits and vegetables and assessing potential health risks. This research aimed to conduct a health risk assessment of presence of pesticide residues in fruits and vegetables in the Kampala Metropolitan Area in Uganda.

\section{Method}

Pesticides were measured in 160 fruits and vegetables samples collected at farms, markets, street vendors, restaurants and homes using liquid chromatography-tandem mass spectrometry and gas chromatography-mass spectrometry. Fruit and vegetable consumption information was collected from 2,177 people. Pesticide concentrations were compared with European Union maximum residual limits (MRLs). Mean values of pesticide concentration residues found in the sample of fruits and vegetables; and fruits and vegetables intake and body weight were used to calculate the estimated daily intake (EDI) of pesticide residues. EDI values were compared with acceptable daily intakes (ADI) to calculate the hazard quotient by age group, and stage at which consumption happens along the chain.

\section{Results}

Overall, 57 pesticides were detected in fruits and vegetables from farm to fork. Of the 57,39 pesticides were detected in all the fruits and vegetables studied. Concentrations of fonofos, fenitrothion and fenhexamid were above the European Union MRLs in some samples. Hazard quotients based on dietary ingestion scenarios for eighteen pesticides, including dichlorvos (444) alanycarb (314), fonofos (68), fenitrothion (62), dioxacarb (55) and benfuracarb (24) and others, were above 1, indicating the possibility of chronic health risk to consumers. Chronic health risk decreased with age but was stable for stage at which consumption happens along the food chain. The number of pesticides with EDI greater than the ADI decreased with increase in age; with $18,13,9,11,8,9$, and 9 pesticides for age groups $<5,5-12,13-19,20-25,36-49$ and $\geq 50$ respectively.

\section{Conclusion}

Chronic dietary pesticide exposures to Ugandans are likely common, and for some pesticides result in exposure exceeding health-based benchmarks. Risks were highest for younger participants. There is an urgent need to increase monitoring and regulation of pesticides in fruits and vegetables in order to protect consumers, especially the children who are especially vulnerable to the adverse effects of pesticides.

\section{Introduction}

Pesticides are widely used in agriculture to control pests and disease in crops to improve the quality of produce [1]. Some commonly used classes of pesticides include organophosphates, carbamates, pyrethroids and neonicotinoids [2-5]. Most of these pesticide chemical groups, such as organophosphates, are broad spectrum insecticides, fungicides or herbicides used to control many different pests, diseases or weeds in different crops [6]. Many organophosphates, carbamates, pyrethroids and neonicotinoids, all neurotoxic pesticides, are registered for use in Uganda, [7] and use is increasing with increasing consumption of fruits and vegetables including tomatoes, cabbage and watermelons, to name but a few [8].

Organophosphates and carbamates pesticides are generally not persistent because they degrade when exposed to sunlight, air and soils, but they often have high solubility and volatility and are heavily used in many farming systems [9]. Organophosphates and carbamates inhibit cholinesterase and may impact neurodevelopment by other mechanisms, including interference in synaptogenesis and myelin sheath formation $[10,11]$. Pyrethroids and neonicotinoids are often systemic pesticides with a higher affinity to soil and, especially for neonicotinoids, have the potential to bioaccumulate. They also have low volatility $[12,13]$. Pyrethroids act by altering the function of voltage-gated sodium channel and consequently disrupt electrical signalling in the nervous system [14] and are generally less acutely toxic than organophosphates [15]. However, they are neurotoxicants and have been associated with confusion, lacrimation and salivation [16] and also poorer development and asthma in children $[17,18]$. The mechanism of toxicity for neonicotinoids is based on selective binding and interaction with nicotinic acetylcholine receptor sites of a target organism causing paralysis that leads to death [19-21], and they have also been associated with development or neurological disorders [22] in humans. 
The use of these chemicals in agriculture may result in residues in food and expose consumers. Events where high levels of pesticide contamination has occurred has resulted in acute health risks including nausea, excessive sweating and salivation, diarrhoea and vomiting, inhibition of blood clotting, and paralysis of the respiratory and circulatory systems [23]. Several studies have shown that chronic exposure to low levels of some neurotoxic pesticides are associated with poorer learning and behavioral problems in children, memory loss, loss of coordination, reduced speed of response to stimuli, reduced visual ability, altered or uncontrolled mood and general weakness; reproductive defects and cancers [24-26].

In Uganda, the volume of pesticides used has increased from 338 tonnes in the 1960s to 82,560.29 tonnes in 2019 [27]. Many farmers do not follow recommended mixing concentrations on label instructions and pre-harvest intervals [28]. Such improper pesticide use practices may result in higher levels of pesticide residues in fruits and vegetables [29] that leave the farm to the final consumer. While they are important sources of minerals, vitamins, and other healthful nutrients, consumption of fruits and vegetables contaminated with pesticide can be a route of exposure to hazardous chemicals. Fruit and vegetable consumption is a protective factor for noncommunicable diseases such as diabetes [30], and consumption is rising in among Ugandans, which consume an average 260 grams of fruits and vegetables each day [31]. Fruit and vegetable consumption has grown among residents of the Kampala Metropolitan Area (KMA) [32], and organophosphate, carbamate, pyrethroid and neonicotinoid pesticides have been previously detected in the tomatoes, watermelon, cabbages among others in this market [28, $33,34]$.

In the current study, we assessed potential pesticide exposures and health risks from consumption of fruit and vegetables by residents of the KMA, in Uganda.

\section{Materials And Methods Study area}

This study was conducted in Kampala, Wakiso and Mukono Districts, three of the 5 districts that make up the KMA in Uganda. The 3 districts have a population of $10,812,700$ people [35] and cover an area of $1,000 \mathrm{~km}^{2}$ [36]. Agriculture is the largest economic activity in Central Uganda within which the KMA is located, supporting $39.3 \%$ of the population [35]. This region has many large fresh produce markets, restaurants, fruit and vegetable vending along the streets, as well as many of the farms where fruits and vegetables consumed within central Uganda are grown. Kampala, Wakiso and Mukono are inhabited by $15 \%$ of Uganda's population and contain Uganda's districts that consume a large volume of the fruit and vegetables produced.

Ethical clearance to conduct the study was obtained from the Makerere University School of Public Health Higher Degrees, Research and Ethics Committee; and registered by Uganda National Council for Science and Technology (SS 5203). Participation in the study was voluntary and participants (farmers, restaurants market managers, street fruit and vegetable vendors, and household heads) provided informed written consent to collect samples and fruit and vegetable dietary intake information. All samples and questionnaire were coded with an anonymous identification number.

\section{Pesticide residue data}

\section{Sampling of fruits and vegetables}

Fruits and vegetables samples were collected from key stages along the supply chain including farms (50), markets (50), street vendors (20), restaurants (20) and homes (20), totaling 160 samples. The detailed methodology used to collect the fruits and vegetable samples has been previously described [33]. Briefly, fresh fruit and vegetable samples were purchased and collected in sterile polythene bags or PET (polyethylene terephthalate) plastic containers from selected farms, markets, and street vendors. Samples of ready-to-eat foods were bought from restaurants and homes, especially juices and salads that do not contain fat-soluble substances. Three replicate fruit and vegetable samples were collected at each location measuring at least $1 \mathrm{~kg}$ for larger produce as suggested by Codex guidelines [37, 38]; processed food samples were at least 1 $\mathrm{kg}$ or 1 litre in case of juice. The samples were stored in a cooler and transported to the laboratory within 8 hours and stored at $-20^{\circ} \mathrm{C}$ until analysis.

\section{Sample preparation and extraction}

A total of 93 pesticides residues were screened in the fruit and vegetable samples (Supplementary Table 1). Using the Quick, Easy, Cheap, Effective, Rugged and Safe (QuEChERS) approach, samples were prepared, cleaned and extracted to determine of pesticide residues [39]. Briefly, 1-2 kgs of each sample was chopped, grinded and blended to homogenize the sample. Of the homogenized sample, $200 \mathrm{~g}$ put into containers and immediately frozen in order to minimize the risk of degradation of any pesticide residues present. Ten grams of homogenized sample was mixed with $3 \mathrm{~g}$ of sodium bicarbonate $\left(\mathrm{NaHCO}_{3}\right)$ and $20.0 \mathrm{~mL}$ ethyl acetate and centrifuged. The samples were then vortexed and placed on a mechanical shaker at $300 \mathrm{rpm} / \mathrm{min}$ for 15 minutes; $10 \mathrm{~g}$ of anhydrous sodium sulphate $\left(\mathrm{Na}_{2} \mathrm{SO}_{4}\right)$ was then added and vortexed 
and centrifuged. We filtered the crude extract using a 0.2-micron PTFE syringe filter. The final supernatant layer $(0.50 \mathrm{~g} / \mathrm{mL}) \mathrm{was}$ transferred into the vials and injected into the LC-MS/MS for analysis of pesticide residues [33].

\section{Pesticide analysis}

Liquid chromatography - Tandem mass spectrometry (LC-MS/MS) analysis was carried to detect and ensure quality of the pesticides residue measurements. A zorbax eclipse plus C18 capillary column ( $150 \mathrm{~mm}$ with $2.1 \mathrm{~mm}$ internal diameter and $1.8 \mu \mathrm{m}$ particle size) operating at $35^{\circ} \mathrm{C}$ to $360^{\circ} \mathrm{C}$ was used with the internal temperature set at $35^{\circ} \mathrm{C}$ for 1 minute, then ramped to $120^{\circ} \mathrm{C}$ per minute and $375^{\circ} \mathrm{C}$ per minute. This process was run over two mobile phases. Phase A involved - water ( $0.1 \%$ formic acid, $5 \mathrm{mM}$ ammonium formate, and $2 \% \mathrm{MeOH})$. $\mathrm{Phase} \mathrm{B}$ involved methanol ( $0.1 \%$ formic acid, $5 \mathrm{mM}$ ammonium formate, $2 \%$ water). The injector temperature was $120^{\circ} \mathrm{C}$ and carrier gas was helium at a flow rate of $13 \mathrm{~L} /$ minute with splitless injection. The injection volume was $5 \mu \mathrm{L}$ at a pressure of 45 psi. The MS ion source temperature was $120^{\circ} \mathrm{C}$ for a minute and raised at a rate of $35^{\circ} \mathrm{C}$ per minute to $375^{\circ} \mathrm{C}$. Confirmation analysis utilised LC-MS/MS which requires two product ions. Compounds with only one product ion were quantified and confirmed using the second ion. For confirmation, the relative ion intensity for a pesticide in a sample was calculated and the value compared to the equivalence for a calibration standard. For positive confirmation, the retention times were matched to the calibration standard as well the relative ion intensities according to the recommended maximum tolerances. Limits of detection (LOD) was determined during the method validation and measurements of uncertainty.

The method developed by Keppel et al. at the United States Food and Drug Administration (U.S. FDA) [32, 33] was used to measure dithiocarbamates (mancozeb, maneb, dithane, thiram, metam sodium and propineb. Frozen sub-samples of $10 \mathrm{~g}$ were placed into a Duran bottle $(250 \mathrm{ml})$ and mixed with isooctane $(20 \mathrm{ml})$ followed by stannous chloride (reducing solution) in hydrochloric acid (100ml), and sealed immediately with a septum and cap. The sample was incubated at $80^{\circ} \mathrm{C}$ in a water bath for 1.5 hours with frequent shaking. The Duran bottles were removed and left at ambient for approximately 1 hour. The bottles were frozen for 30 minutes to allow the generated carbon disulphide gas to condense. The samples were shaken and left for 5 minutes. The organic phase (iso-octane) was removed and placed in a vial prior to the quantitation of carbon disulphide by Gas Chromatography-Mass spectrometry (GC-MS). Spiking was done twice, once at the limit of quantitation (LOQ) $(50 \mu \mathrm{g} / \mathrm{kg})$ and another at the expected residue level (1000 $\mu \mathrm{g} / \mathrm{kg})$, as obtained from previous runs during instrument optimization. A 5-point calibration was used, ranging from $0.125-5 \mu \mathrm{g} / \mathrm{ml}$. The method's LOQ was set at $0.00005 \mathrm{mg} / \mathrm{kg}$ which equates to the calibration standard of $0.125 \mu \mathrm{g} / \mathrm{ml}$. All extracts were analyzed using GC-MS. Final pesticide residues concentrations were expressed in mg/kg of food.

\section{Dietary consumption data}

A modified semi-structured food frequency questionnaire from the World Health Organization's (WHO) STEPwise approach to surveillance, standardized method of collecting data on risk factors for noncommunicable diseases (NCDs) [40] was used was used to interview 2,177 participants to assess fruit and vegetable consumption over a 24-hour dietary recall period and their body weight was concurrently measured using a weighing scale. The detailed methodology on this has been described elsewhere [31]. Briefly, based on typical Ugandan diets, a food album was developed with different quantities of selected fruits and vegetables. Each research assistant was given a copy of the food album as a guide during the interview. Respondents were asked to identify the quantities they consume per serving to determine the amounts consumed. Based on portion size in the food album, we estimated intake in grams of each fruit and vegetable each day of the week. For children under 18 years, their parents or caretakers were interviewed. Participant's weight was also measured thrice and the average calculated. For children below 2 years who cannot stand, their weight was obtained by reviewing their immunization chart or asking their parents or caregivers the measurement from their last weighing event. Socio-demographics data was also obtained using the study questionnaire. Consumption data was collected for five commonly consumed and pesticide intensive fruits and vegetables, that is: watermelon, passion fruit, tomato, cabbage and eggplant following interviews with farmers and agricultural extension. workers

Health risk assessment

We first prepared descriptive statistics of the pesticide residue levels in the produce samples. The mean pesticide concentrations were then compared with European Union maximum residual limits (EU MRLs) obtained from the pesticide residue database (https://ec.europa.eu/food/plant/pesticides/eu-pesticides-database/mrls/?event=search.pr) [41]. EU MRLs were used because they provided comprehensive standard values for all fruits studied; they have also been used in other African studies [42, 43]. We also used the mean pesticide concentrations to estimated pesticide intake to compare with acceptable daily intakes (ADIs). Estimated daily intake (mg/kg/bw/day) was calculated by multiplying the mean concentration of each pesticide (C) and the fruits and vegetable consumption rate (FVCR) (g/day) and dividing this by body weight (BW) using the following formula EDI = (C x FVCR)/BW $[44,45]$. The FVCR used was obtained from the dietary consumption survey published in Plos One [31]. FVCR was calculated as mean consumption of fruits and vegetables studied for the sample population as well as for different age groups studied that is $<5,5-12,13-19,20-24,25-35,36-49$ and $50+$. BW used was measured during the dietary consumption survey with the mean for general sample population and age groups calculated accordingly. The acceptable daily intake (ADI) (mg/kg/bw/day) for the different pesticides was obtained from the EU pesticide residue database [41]. The chronic risk assessment for pesticide residue was calculated by comparing EDI with the ADI to get the hazard quotient (HQ) using the following equation;

Page 4/19 
$\mathrm{HQ}=\mathrm{EDI} / \mathrm{ADI}$. A hazard quotient $(\mathrm{HQ})>1$ indicates exposures over the health-based benchmarks and the potential to induce unacceptable health risks among consumers.

\section{Results}

\section{Pesticide residue concentrations}

The mean concentration of organophosphates, carbamates, pyrethroids and neonicotinoids among other pesticides detected in watermelons, passion fruit, tomato, cabbage and eggplants and comparisons with their respective MRLs are shown in Table 1. Out of the 62 pesticide active ingredients detected, 5 were excluded due to the lack of verified maximum residue levels (MRLs) in the EU database for the studied fruits and vegetables. Therefore, 57 pesticides were considered for the risk assessment. Of the 57 pesticides, 39 pesticides were detected in all the fruits and vegetable types. Dimethoate was detected only in watermelon with a mean concentration of $0.0007 \mathrm{mg} / \mathrm{kg}$. Fonofos was detected in all fruits and vegetables with concentrations above the MRLs in watermelon (0.03 mgkg), passion fruit $(0.02 \mathrm{mg} / \mathrm{kg}), \mathrm{cabbages}(0.11 \mathrm{mg} / \mathrm{kg})$ and eggplants $(0.14 \mathrm{mg} / \mathrm{kg})$. Methidathion was not detected in watermelon and cabbages and malathion was not detected in passion fruit.

Methacrifos was detected in passion fruit at $0.00003 \mathrm{mg} / \mathrm{kg}$ and cabbages at $0.000002 \mathrm{mg} / \mathrm{kg}$. Ethoprophos was not detected in vegetables but only in eggplants at $0.0003 \mathrm{mg} / \mathrm{kg}$. Coumaphos and pirimiphos-methyl were detected only cabbages at $0.0000005 \mathrm{mg} / \mathrm{kg}$ and eggplants at $0.00001 \mathrm{mg} / \mathrm{kg}$ respectively. Apart from passion fruit, fenitrothion concentration was above the MRLs in watermelon (0.02 $\mathrm{mg} / \mathrm{kg}$ ), tomato $(0.013 \mathrm{mg} / \mathrm{kg})$, cabbage $(0.03 \mathrm{mg} / \mathrm{kg})$ and eggplant $(0.03 \mathrm{mg} / \mathrm{kg})$. Neonicotinoids were detected in almost all fruits and vegetables apart from thiacloprid that was only detected in passion fruit $0.000007 \mathrm{mg} / \mathrm{kg}$ and tomato $0.000002 \mathrm{mg} / \mathrm{kg}$. Deltamethrin, azoxystrobin and proquinazid were only detected in vegetables with concentrations below the MRLs. Although not detected in tomato, fenhexamid's concentration was above the MRLs in watermelon $(0.01 \mathrm{mg} / \mathrm{kg})$, passion fruit $(0.07 \mathrm{mg} / \mathrm{kg})$ and cabbage $(0.03 \mathrm{mg} / \mathrm{kg})$. 
Table 1

Concentration of pesticide residues per fruit and vegetable type compared with the MRLs

\begin{tabular}{|c|c|c|c|c|c|c|c|c|c|c|c|}
\hline \multirow[t]{2}{*}{$\begin{array}{l}\text { Pesticide } \\
\text { residues }\end{array}$} & \multirow[t]{2}{*}{$\begin{array}{l}\text { LOD } \\
(\mathrm{mg} / \mathrm{kg})\end{array}$} & \multicolumn{2}{|c|}{$\begin{array}{l}\text { Water melon } \\
(\mathrm{mg} / \mathrm{kg})\end{array}$} & \multicolumn{2}{|c|}{$\begin{array}{l}\text { Passion fruit } \\
\text { (mg/kg) }\end{array}$} & \multicolumn{2}{|c|}{ Tomato $(\mathrm{mg} / \mathrm{kg})$} & \multicolumn{2}{|c|}{ Cabbage $(\mathrm{mg} / \mathrm{kg})$} & \multicolumn{2}{|c|}{ Eggplant (mg/kg) } \\
\hline & & Mean & MRL & Mean & MRL & Mean & MRL & Mean & MRL & Mean & MRL \\
\hline Dithiocarbamate ${ }^{a}$ & 0.000006 & 0.001 & 1.5 & 0.00007 & 0.05 & 0.0002 & 3 & 0.0006 & 3 & 0.0004 & 3 \\
\hline Omethoate & 0.00002 & 0.0004 & 0.01 & 0.0002 & 0.01 & 0.0003 & 0.01 & $\mathrm{BDL}$ & 0.01 & 0.00007 & 0.01 \\
\hline Acephate & 0.00003 & 0.001 & 0.01 & 0.001 & 0.01 & 0.0001 & 0.01 & 0.00008 & 0.01 & 0.0002 & 0.01 \\
\hline Monocrotophos & 0.00001 & 0.00003 & 0.01 & 0.00004 & 0.01 & $\mathrm{BDL}$ & 0.01 & 0.00003 & 0.01 & 0.00002 & 0.01 \\
\hline Vamidothion & 0.00001 & $\mathrm{BDL}$ & 0.01 & $\mathrm{BDL}$ & 0.01 & $\mathrm{BDL}$ & 0.01 & $\mathrm{BDL}$ & 0.01 & 0.00008 & 0.01 \\
\hline Dimethoate & 0.000008 & 0.0007 & 0.01 & $\mathrm{BDL}$ & 0.01 & $\mathrm{BDL}$ & 0.01 & $\mathrm{BDL}$ & 0.01 & $\mathrm{BDL}$ & 0.01 \\
\hline Mevinphos & 0.00003 & $\mathrm{BDL}$ & 0.01 & $\mathrm{BDL}$ & 0.01 & $\mathrm{BDL}$ & 0.01 & 0.00004 & 0.01 & 0.00005 & 0.01 \\
\hline Phosphamidon & 0.00002 & $\mathrm{BDL}$ & 0.01 & $\mathrm{BDL}$ & 0.01 & $\mathrm{BDL}$ & 0.01 & $\mathrm{BDL}$ & 0.01 & 0.00005 & 0.01 \\
\hline Fonofos & 0.00001 & $0.03^{*}$ & 0.01 & $0.2^{*}$ & 0.01 & 0.2 & 10 & $0.1^{*}$ & 0.01 & $0.1 *$ & 0.01 \\
\hline Azamethiphos & 0.000005 & 0.00002 & 0.01 & 0.000007 & 0.01 & $\mathrm{BDL}$ & 0.01 & $\mathrm{BDL}$ & 0.01 & $\mathrm{BDL}$ & 0.01 \\
\hline Dichlorvos & 0.00002 & 0.003 & 0.01 & 0.007 & 0.01 & 0.0001 & 0.01 & 0.0002 & 0.01 & 0.0007 & 0.01 \\
\hline Malaoxon & 0.00001 & $\mathrm{BDL}$ & 0.02 & $\mathrm{BDL}$ & 0.02 & 0.00004 & 0.02 & 0.00002 & 0.02 & $\mathrm{BDL}$ & 0.02 \\
\hline Methidathion & 0.00001 & $\mathrm{BDL}$ & 0.02 & 0.000003 & 0.02 & 0.00002 & 0.02 & $\mathrm{BDL}$ & 0.02 & 0.000001 & 0.02 \\
\hline Malathion & 0.00002 & $\mathrm{BDL}$ & 0.02 & $\mathrm{BDL}$ & 0.02 & $\mathrm{BDL}$ & 0.02 & $\mathrm{BDL}$ & 0.02 & 0.00007 & 0.02 \\
\hline Methacrifos & 0.000005 & $\mathrm{BDL}$ & 0.01 & 0.00003 & 0.01 & $\mathrm{BDL}$ & 0.01 & $\mathrm{BDL}$ & 0.01 & $\mathrm{BDL}$ & 0.01 \\
\hline Ethoprophos & 0.00008 & $\mathrm{BDL}$ & 0.01 & $\mathrm{BDL}$ & 0.01 & $\mathrm{BDL}$ & 0.01 & $\mathrm{BDL}$ & 0.01 & $\mathrm{BDL}$ & 0.01 \\
\hline Fenamiphos & 0.000009 & $\mathrm{BDL}$ & 0.02 & $\mathrm{BDL}$ & 0.02 & $\mathrm{BDL}$ & 0.04 & $\mathrm{BDL}$ & 0.04 & $\mathrm{BDL}$ & 0.02 \\
\hline Quinalphos & 0.00003 & 0.0001 & 0.01 & 0.0001 & 0.01 & 0.00003 & 0.01 & $\mathrm{BDL}$ & 0.01 & 0.0001 & 0.01 \\
\hline Coumaphos & 0.00002 & $\mathrm{BDL}$ & 0.01 & $\mathrm{BDL}$ & 0.01 & $\mathrm{BDL}$ & 0.01 & $\mathrm{BDL}$ & 0.01 & $\mathrm{BDL}$ & 0.01 \\
\hline $\begin{array}{l}\text { Chlorpyriphos- } \\
\text { methyl }\end{array}$ & 0.000008 & $\mathrm{BDL}$ & 0.01 & 0.00004 & 0.01 & 0.00009 & 0.01 & 0.00005 & 0.01 & 0.00002 & 0.01 \\
\hline Temephos & 0.000008 & $\mathrm{BDL}$ & 0.01 & 0.00001 & 0.01 & $\mathrm{BDL}$ & 0.01 & 0.000009 & 0.01 & $\mathrm{BDL}$ & 0.01 \\
\hline Profenofos & 0.00001 & 0.003 & 0.01 & 0.00002 & 0.01 & 0.04 & 10 & 0.003 & 0.01 & 0.005 & 0.01 \\
\hline Pirimiphosmethyl & 0.00002 & $\mathrm{BDL}$ & 0.01 & $\mathrm{BDL}$ & 0.01 & $\mathrm{BDL}$ & 0.01 & $\mathrm{BDL}$ & 0.01 & 0.00001 & 0.01 \\
\hline Fenitrothion & 0.00001 & $0.02 *$ & 0.01 & 0.004 & 0.01 & $0.01 *$ & 0.01 & $0.03^{*}$ & 0.01 & $0.03^{*}$ & 0.01 \\
\hline Aminocarb & 0.00002 & $\mathrm{BDL}$ & 0.01 & 0.0007 & 0.01 & $\mathrm{BDL}$ & 0.01 & 0.00008 & 0.01 & 0.00002 & 0.01 \\
\hline Methomyl & 0.00003 & 0.00006 & 0.015 & 0.00003 & 0.01 & 0.00003 & 0.01 & $\mathrm{BDL}$ & 0.01 & 0.00003 & 0.01 \\
\hline Aldicarbfragment & 0.00001 & 0.00002 & 0.02 & 0.00002 & 0.02 & 0.00004 & 0.02 & 0.00002 & 0.02 & $\mathrm{BDL}$ & 0.02 \\
\hline Pirimicarb & 0.00003 & 0.00004 & 0.5 & 0.00004 & 0.01 & $\mathrm{BDL}$ & 0.5 & $\mathrm{BDL}$ & 0.5 & $\mathrm{BDL}$ & 0.5 \\
\hline Dioxacarb & 0.00001 & 0.004 & 0.01 & 0.003 & 0.01 & 0.003 & 0.01 & 0.003 & 0.01 & 0.004 & 0.01 \\
\hline Carbaryl & 0.000008 & $\mathrm{BDL}$ & 0.01 & $\mathrm{BDL}$ & 0.01 & $\mathrm{BDL}$ & 0.01 & $\mathrm{BDL}$ & 0.01 & 0.00001 & 0.01 \\
\hline Carbofuran & 0.000009 & 0.00003 & 0.01 & $\mathrm{BDL}$ & 0.01 & $\mathrm{BDL}$ & 0.002 & $\mathrm{BDL}$ & 0.002 & 0.00003 & 0.002 \\
\hline Alanycarb & 0.00001 & 0.0001 & 0.02 & 0.00006 & 0.02 & 0.08 & 0.02 & 0.01 & 0.02 & 0.006 & 0.02 \\
\hline Benfuracarb & 0.00005 & 0.0005 & 0.01 & $\mathrm{BDL}$ & 0.01 & 0.004 & 0.002 & 0.07 & 0.002 & 0.004 & 0.002 \\
\hline Methiocarb & 0.00004 & $\mathrm{BDL}$ & 0.03 & $\mathrm{BDL}$ & 0.03 & BDL & 0.03 & 0.00005 & 0.03 & BDL & 0.03 \\
\hline
\end{tabular}




\begin{tabular}{|c|c|c|c|c|c|c|c|c|c|c|c|}
\hline \multirow[t]{2}{*}{$\begin{array}{l}\text { Pesticide } \\
\text { residues }\end{array}$} & \multirow[t]{2}{*}{$\begin{array}{l}\text { LOD } \\
(\mathrm{mg} / \mathrm{kg})\end{array}$} & \multicolumn{2}{|c|}{$\begin{array}{l}\text { Water melon } \\
(\mathrm{mg} / \mathrm{kg})\end{array}$} & \multicolumn{2}{|c|}{$\begin{array}{l}\text { Passion fruit } \\
\text { (mg/kg) }\end{array}$} & \multicolumn{2}{|c|}{ Tomato $(\mathrm{mg} / \mathrm{kg})$} & \multicolumn{2}{|c|}{ Cabbage (mg/kg) } & \multicolumn{2}{|c|}{ Eggplant (mg/kg) } \\
\hline & & Mean & MRL & Mean & MRL & Mean & MRL & Mean & MRL & Mean & MRL \\
\hline Imidacloprid & 0.00003 & 0.0007 & 0.2 & 0.0008 & 0.05 & 0.0004 & 0.5 & 0.0004 & 0.5 & 0.0002 & 0.5 \\
\hline Acetamiprid & 0.00002 & 0.004 & 0.2 & 0.002 & 0.01 & 0.008 & 0.5 & 0.005 & 0.4 & 0.001 & 0.2 \\
\hline Thiacloprid & 0.00001 & $\mathrm{BDL}$ & 0.2 & $\mathrm{BDL}$ & 0.01 & $\mathrm{BDL}$ & 0.5 & $\mathrm{BDL}$ & 0.3 & $\mathrm{BDL}$ & 0.7 \\
\hline Bifenthrin & 0.00002 & 0.0001 & 0.01 & 0.00004 & 0.01 & 0.0004 & 0.3 & 0.00005 & 0.4 & $\mathrm{BDL}$ & 0.3 \\
\hline $\begin{array}{l}\text { Lambda- } \\
\text { Cyhalothrin }\end{array}$ & 0.00002 & 0.0002 & 0.06 & 0.0001 & 0.01 & 0.0002 & 0.07 & 0.0002 & 0.15 & 0.0002 & 0.3 \\
\hline Deltamethrin & 0.00001 & $\mathrm{BDL}$ & 0.02 & $\mathrm{BDL}$ & 0.01 & $\mathrm{BDL}$ & 0.07 & 0.00006 & 0.1 & $\mathrm{BDL}$ & 0.4 \\
\hline Cypermethrin & 0.00001 & 0.0002 & 0.2 & $\mathrm{BDL}$ & 0.05 & 0.001 & 0.5 & 0.0004 & 1 & 0.0004 & 0.5 \\
\hline Carbendazim & 0.00002 & $\mathrm{BDL}$ & 0.1 & 0.0001 & 0.1 & $\mathrm{BDL}$ & 0.3 & 0.0001 & 0.1 & $\mathrm{BDL}$ & 0.5 \\
\hline Imazalil & 0.00001 & 0.0005 & 0.01 & 0.0004 & 0.01 & 0.0001 & 0.3 & 0.0003 & 0.01 & 0.0003 & 0.01 \\
\hline Metazachlor & 0.00001 & 0.00001 & 0.02 & 0.00004 & 0.02 & 0.00006 & 0.02 & 0.00002 & 0.4 & 0.00002 & 0.02 \\
\hline Metalaxyl & 0.00002 & $\mathrm{BDL}$ & 0.2 & $\mathrm{BDL}$ & 0.01 & 0.00005 & 0.3 & $\mathrm{BDL}$ & 0.06 & $\mathrm{BDL}$ & 0.01 \\
\hline Azaconazole & 0.000006 & 0.000009 & 0.01 & 0.0001 & 0.01 & 0.000008 & 0.01 & 0.00007 & 0.01 & 0.000008 & 0.01 \\
\hline Clomazone & 0.000007 & $\mathrm{BDL}$ & 0.01 & $\mathrm{BDL}$ & 0.01 & $\mathrm{BDL}$ & 0.01 & $\mathrm{BDL}$ & 0.01 & $\mathrm{BDL}$ & 0.01 \\
\hline Azoxystrobin & 0.000007 & $\mathrm{BDL}$ & 1 & $\mathrm{BDL}$ & 4 & 0.00456 & 3 & 0.003 & 5 & 0.003 & 3 \\
\hline Pyrimethanil & 0.00002 & 0.0001 & 0.01 & 0.00008 & 0.01 & 0.0001 & 1 & 0.00008 & 0.01 & 0.00006 & 1 \\
\hline Spirotetramat & 0.00002 & 0.00003 & 0.2 & $\mathrm{BDL}$ & 0.1 & 0.00009 & 2 & 0.00001 & 2 & $\mathrm{BDL}$ & 2 \\
\hline Fenhexamid & 0.00001 & $0.01 *$ & 0.01 & $0.07^{*}$ & 0.01 & $\mathrm{BDL}$ & 2 & $0.03^{*}$ & 0.01 & 0.009 & 2 \\
\hline Fenarimol & 0.00001 & 0.0006 & 0.05 & 0.0003 & 0.02 & 0.0002 & 0.02 & 0.0003 & 0.02 & 0.0004 & 0.02 \\
\hline Fluazifop & 0.00002 & 0.005 & 0.01 & $\mathrm{BDL}$ & 0.01 & $\mathrm{BDL}$ & 0.06 & 0.0004 & 0.01 & $\mathrm{BDL}$ & 1 \\
\hline Flufenoxuron & 0.00002 & $\mathrm{BDL}$ & 0.01 & $\mathrm{BDL}$ & 0.01 & $\mathrm{BDL}$ & 0.01 & $\mathrm{BDL}$ & 0.01 & $\mathrm{BDL}$ & 0.01 \\
\hline Pyriproxyfen & 0.000007 & $\mathrm{BDL}$ & 0.05 & $\mathrm{BDL}$ & 0.05 & $\mathrm{BDL}$ & 1 & $\mathrm{BDL}$ & 0.05 & $\mathrm{BDL}$ & 1 \\
\hline Quinoxyfen & 0.00003 & $\mathrm{BDL}$ & 0.05 & 0.00005 & 0.02 & 0.00004 & 0.02 & $\mathrm{BDL}$ & 0.02 & 0.00003 & 0.02 \\
\hline Proquinazid & 0.00001 & $\mathrm{BDL}$ & 0.02 & $\mathrm{BDL}$ & 0.02 & 0.001 & 0.15 & 0.0003 & 0.02 & 0.00009 & 0.02 \\
\hline
\end{tabular}

Health risk assessment by stage of consumption along the chain

The risk of exposures to pesticides residues in fruits and vegetables are evaluated by the stage at which consumption may occur along the chain including at the farm, market, street vendor, restaurant and home as shown in Table 2. The EDI was higher than the ADI in at least one of the stages at which consumption may occur in 16 of the 57 pesticides assessed. EDIs for dichlorvos, fenitrothion, alanycarb and benfuracarb were above the $\mathrm{ADI}$ at all stages of consumption. EDIs for fonofos and profenofos exceeded the ADI at four stages of consumption. Fonofos, dichlorvos, fenitrothion, dioxacarb, alanycarb and benfuracarb presented the highest risk levels with HQs of 27.5, 442.6, 23.6, 29.5, 118.0 and 23.6 respectively, at the farm and throughout the entire supply chain (See supplementary table 2). Overall, pesticide concentration at street vendors presented lower HQs and consequently lower likelihood for health risks compared to other stages along the chain (Fig. 1). 
Table 2

Estimated daily intake (mgkg/bw/day) for fruits and vegetables by stage along the chain

\begin{tabular}{|c|c|c|c|c|c|c|}
\hline \multirow[t]{2}{*}{ Pesticides } & \multirow[t]{2}{*}{ ADI (mg/kg/bw/day) } & \multicolumn{5}{|c|}{ EDI (mg/kg/bw/day) } \\
\hline & & Farm & Market & Street & Restaurant & Home \\
\hline Dithiocarbamate $^{a}$ & 0.05 & 0.002 & 0.003 & 0.005 & 0.002 & 0.002 \\
\hline Omethoate & 0.002 & 4.7E-06 & $0.002 *$ & BDL & $0.002 *$ & $2.9 \mathrm{E}-06$ \\
\hline Acephate & 0.03 & 0.005 & 0.004 & 0.0004 & 0.002 & 0.0006 \\
\hline Monocrotophos & 0.0006 & 0.0001 & 0.0002 & 0.0002 & 0.0002 & 0.0001 \\
\hline Vamidothion & 0.008 & 0.0002 & 0.0002 & $5.9 \mathrm{E}-07$ & $3.54 \mathrm{E}-06$ & $2.95 \mathrm{E}-06$ \\
\hline Dimethoate & 0.002 & BDL & $0.003^{*}$ & BDL & BDL & BDL \\
\hline Mevinphos & 0.001 & 0.0002 & 0.0002 & $2.4 \mathrm{E}-06$ & $2.4 \mathrm{E}-06$ & $5.9 \mathrm{E}-05$ \\
\hline Phosphamidon & 0.0005 & 1.2E-06 & 1.18E-06 & $1.18 \mathrm{E}-06$ & BDL & 1.77E-06 \\
\hline Fonofos & 0.03 & $0.8^{*}$ & $0.9 *$ & 0.006 & $1.2^{*}$ & $0.4^{*}$ \\
\hline Azamethiphos & 0.025 & 2.4E-06 & 5.9E-05 & $1.2 \mathrm{E}-06$ & $5.9 \mathrm{E}-06$ & $\mathrm{BDL}$ \\
\hline Dichlorvos & 0.00008 & $0.04 *$ & $0.0006^{*}$ & $0.0004^{*}$ & $0.002^{*}$ & $0.003^{*}$ \\
\hline Malaoxon & 0.03 & 4.1E-06 & 4.1E-06 & 0.0003 & $5.9 \mathrm{E}-05$ & 5.9E-05 \\
\hline Methidathion & 0.001 & $\mathrm{BDL}$ & $\mathrm{BDL}$ & $1.2 \mathrm{E}-06$ & BDL & 0.0002 \\
\hline Malathion & 0.03 & 0.0003 & $\mathrm{BDL}$ & $\mathrm{BDL}$ & $\mathrm{BDL}$ & $2.9 \mathrm{E}-06$ \\
\hline Methacrifos & 0.006 & BDL & 0.0001 & $\mathrm{BDL}$ & BDL & BDL \\
\hline Ethoprophos & 0.0004 & $\mathrm{BDL}$ & 0.0001 & $\mathrm{BDL}$ & 0.0001 & 0.0002 \\
\hline Fenamiphos & 0.0008 & 5.9E-08 & $1.2 \mathrm{E}-06$ & 5.9E-05 & $1.8 \mathrm{E}-06$ & BDL \\
\hline Quinalphos & 0.001 & 0.0001 & 0.0005 & 0.0004 & $0.001 *$ & 0.0006 \\
\hline Coumaphos & 0.001 & $1.8 \mathrm{E}-07$ & $\mathrm{BDL}$ & $\mathrm{BDL}$ & BDL & BDL \\
\hline Chlorpyriphos-methyl & 0.01 & 0.0005 & $\mathrm{BDL}$ & 2.9E-06 & 0.0006 & $\mathrm{BDL}$ \\
\hline Temephos & 0.001 & BDL & $3.5 \mathrm{E}-06$ & BDL & $2.9 \mathrm{E}-06$ & 0.0001 \\
\hline Profenofos & 0.03 & $0.1 *$ & $0.04 *$ & $0.04 *$ & $0.06 *$ & 0.004 \\
\hline Pirimiphosmethyl & 0.03 & BDL & 4.7E-06 & $\mathrm{BDL}$ & BDL & BDL \\
\hline Fenitrothion & 0.005 & $0.1 *$ & $0.05 *$ & $0.2^{*}$ & $0.2^{*}$ & $0.02 *$ \\
\hline Aminocarb & 0.001 & 0.0002 & $0.003^{*}$ & 0.0001 & 0.0001 & 5.9E-05 \\
\hline Methomyl & 0.0025 & 0.0002 & 0.0003 & 4.1E-06 & 0.0002 & $2.9 \mathrm{E}-06$ \\
\hline Aldicarbfragment & 0.001 & 0.0001 & 0.0001 & 0.000177 & $5.9 \mathrm{E}-05$ & 0.0001 \\
\hline Pirimicarb & 0.035 & 0.0001 & 0.0001 & $\mathrm{BDL}$ & 0.0004 & 5.9E-05 \\
\hline Dioxacarb & 0.001 & $0.03^{*}$ & $0.02 *$ & $0.02 *$ & BDL & BDL \\
\hline Carbaryl & 0.0075 & 2.4E-06 & $2.4 \mathrm{E}-06$ & $1.8 \mathrm{E}-06$ & BDL & 0.0001 \\
\hline Carbofuran & 0.00015 & 4.1E-06 & $5.9 \mathrm{E}-07$ & $0.0003^{*}$ & $0.0002^{*}$ & $2.9 \mathrm{E}-07$ \\
\hline Alanycarb & 0.001 & $0.1^{*}$ & $0.1^{*}$ & $0.06 *$ & $0.1^{*}$ & $0.2^{*}$ \\
\hline Benfuracarb & 0.01 & $0.2^{*}$ & $0.05^{\star}$ & $0.02 *$ & 4.7E-13 & $0.02 *$ \\
\hline Methiocarb & 0.00025 & 0.0002 & 5.9E-05 & 0.0002 & 4.1E-06 & 5.9E-07 \\
\hline Imidacloprid & 0.06 & 0.003 & 0.002 & 0.005 & 0.006 & 0.001 \\
\hline
\end{tabular}

BDL - Below detection limit, ADI - Acceptable Daily Intake, EDI - Estimated Daily Intake * - EDI greater than ADI (HQ > 1) 


\begin{tabular}{|c|c|c|c|c|c|c|}
\hline \multirow[t]{2}{*}{ Pesticides } & \multirow[t]{2}{*}{ ADI (mg/kg/bw/day) } & \multicolumn{5}{|c|}{ EDI (mg/kg/bw/day) } \\
\hline & & Farm & Market & Street & Restaurant & Home \\
\hline Acetamiprid & 0.025 & $0.04 *$ & 0.02 & 0.01 & 0.01 & 0.005 \\
\hline Thiacloprid & 0.01 & $3.5 \mathrm{E}-06$ & $\mathrm{BDL}$ & $\mathrm{BDL}$ & $\mathrm{BDL}$ & $\mathrm{BDL}$ \\
\hline Bifenthrin & 0.015 & 0.0006 & 0.002 & 4.7E-06 & 0.0002 & $2.4 \mathrm{E}-06$ \\
\hline Lambda-Cyhalothrin & 0.0012 & 0.001 & 0.001 & 0.001 & 0.0006 & 0.0006 \\
\hline Deltamethrin & 0.01 & $2.4 \mathrm{E}-06$ & 0.0002 & $\mathrm{BDL}$ & $\mathrm{BDL}$ & $\mathrm{BDL}$ \\
\hline Cypermethrin & 0.0016 & $0.004^{*}$ & $0.002^{\star}$ & $0.005^{\star}$ & 0.001 & 0.0006 \\
\hline Carbendazim & 0.02 & $1.2 \mathrm{E}-06$ & 0.001 & $2.4 \mathrm{E}-06$ & $5.9 \mathrm{E}-05$ & 4.7E-07 \\
\hline Imazalil & 0.025 & 0.002 & 0.001 & 0.004 & 0.002 & 0.002 \\
\hline Metazachlor & 0.08 & 0.0002 & 0.0002 & 0.0002 & 0.0002 & 0.0001 \\
\hline Metalaxyl & 0.08 & $1.2 \mathrm{E}-06$ & 0.0002 & $\mathrm{BDL}$ & $\mathrm{BDL}$ & $\mathrm{BDL}$ \\
\hline Azaconazole & 0.001 & 4.7E-06 & 0.0005 & 0.0004 & $\mathrm{BDL}$ & 0.0006 \\
\hline Clomazone & 0.133 & $1.2 \mathrm{E}-06$ & $\mathrm{BDL}$ & $\mathrm{BDL}$ & $\mathrm{BDL}$ & $\mathrm{BDL}$ \\
\hline Azoxystrobin & 0.2 & 0.02 & $\mathrm{BDL}$ & $\mathrm{BDL}$ & 0.01 & 0.04 \\
\hline Pyrimethanil & 0.17 & 0.0002 & 0.0006 & 0.001 & 0.0005 & 0.0006 \\
\hline Spirotetramat & 0.05 & 0.0003 & 0.0002 & 0.0002 & $2.9 \mathrm{E}-06$ & 0.0001 \\
\hline Fenhexamid & 0.2 & 0.1 & 0.2 & $0.6 *$ & 0.006 & 0.05 \\
\hline Fenarimol & 0.01 & 0.002 & 0.0006 & 0.002 & 0.002 & 0.005 \\
\hline Fluazifop & 0.004 & 0.0006 & $0.02 *$ & $\mathrm{BDL}$ & $\mathrm{BDL}$ & $\mathrm{BDL}$ \\
\hline Flufenoxuron & 0.01 & $1.8 \mathrm{E}-06$ & $2.4 \mathrm{E}-06$ & $1.8 \mathrm{E}-07$ & $1.2 \mathrm{E}-06$ & $4.1 \mathrm{E}-07$ \\
\hline Pyriproxyfen & 0.05 & $\mathrm{BDL}$ & $\mathrm{BDL}$ & $2.9 \mathrm{E}-06$ & $\mathrm{BDL}$ & $\mathrm{BDL}$ \\
\hline Quinoxyfen & 0.2 & 0.0002 & $5.9 \mathrm{E}-05$ & 0.0003 & $1.2 \mathrm{E}-06$ & 0.0003 \\
\hline Proquinazid & 0.01 & 0.003 & 0.0006 & 0.002 & 0.001 & $\mathrm{BDL}$ \\
\hline
\end{tabular}

\section{Health risk assessment by age group}

We evaluated the risk of consumption of pesticide residues by age of consumers as shown in Table 3. EDIs for fonofos, dichlorvos, profenofos, fenitrothion, dioxacarb, alanycarb, benfuracarb, cypermethrin and fluazifop exceeded ADIs throughout all age groups and consequently pose chronic health risks. The number of pesticides with EDIs greater than the ADI decreased with age with $18,13,9,11,8,9$, and 9 for age groups under 5 years, $5-12,13-19,20-25,36-49$ and $50+$ years respectively. Dichlorvos had the highest risk with a HQ of 444 followed by alanycarb (314), Fonofos (68), fenitrothion (62), dioxacarb (55) and benfuracarb (24) among children under 5 with a similar trend across age groups (see supplementary table 2). Overall, $\mathrm{HQ}$ values decreased across age groups with children under 5 presenting highest risks and adults $50+$ having the lowest chronic health risks for the nine pesticides as shown in Fig. 2. 
Table 3

Estimated daily intake (mgkg/bw/day) for fruits and vegetables by age group

\begin{tabular}{|c|c|c|c|c|c|c|c|c|c|}
\hline \multirow[t]{2}{*}{ Pesticides } & \multirow{2}{*}{$\begin{array}{l}\text { ADI (mg/kg } \\
\text { bw/day) }\end{array}$} & \multicolumn{8}{|c|}{ EDI (mgkg/bw/day) } \\
\hline & & $\begin{array}{l}\text { General } \\
\text { population }\end{array}$ & $<5$ & $5-12$ & $13-19$ & $20-24$ & $25-35$ & $36-49$ & $50+$ \\
\hline Dithiocarbamate ${ }^{a}$ & 0.05 & 0.003 & 0.007 & 0.004 & 0.002 & 0.003 & 0.002 & 0.002 & 0.002 \\
\hline Omethoate & 0.002 & 0.001 & $0.003^{*}$ & 0.002 & 0.001 & 0.001 & 0.001 & 0.001 & 0.001 \\
\hline Acephate & 0.03 & 0.003 & 0.008 & 0.005 & 0.003 & 0.003 & 0.003 & 0.002 & 0.003 \\
\hline Monocrotophos & 0.0006 & 0.0002 & 0.0005 & 0.0003 & 0.0004 & 0.0002 & 0.0002 & 0.0001 & 0.0002 \\
\hline Vamidothion & 0.008 & 0.0001 & 0.0003 & 0.0002 & 0.0001 & 0.0001 & 0.0001 & $\begin{array}{l}9.4 \mathrm{E}- \\
05\end{array}$ & 0.0001 \\
\hline Dimethoate & 0.002 & 0.0008 & $0.002^{*}$ & 0.001 & 0.0008 & 0.0009 & 0.0008 & 0.0007 & 0.0007 \\
\hline Mevinphos & 0.001 & 0.0001 & 0.0003 & 0.0002 & 0.0001 & 0.0001 & 0.0001 & $\begin{array}{l}9.4 \mathrm{E}- \\
05\end{array}$ & 0.0001 \\
\hline Phosphamidon & 0.0005 & $5.9 \mathrm{E}-06$ & 1.7E-05 & $\begin{array}{l}9.4 \mathrm{E}- \\
06\end{array}$ & $\begin{array}{l}5.8 \mathrm{E}- \\
06\end{array}$ & $\begin{array}{l}6.7 \mathrm{E}- \\
06\end{array}$ & $\begin{array}{l}5.4 \mathrm{E}- \\
06\end{array}$ & $\begin{array}{l}4.7 \mathrm{E}- \\
06\end{array}$ & $\begin{array}{l}5.1 \mathrm{E}- \\
06\end{array}$ \\
\hline Fonofos & 0.03 & $0.7^{*}$ & $2.0^{*}$ & $1.2^{*}$ & $0.7^{*}$ & $0.8^{*}$ & $0.8^{*}$ & $0.6^{*}$ & $0.6^{*}$ \\
\hline Azamethiphos & 0.025 & 4.1E-05 & 0.0001 & $\begin{array}{l}6.6 \mathrm{E}- \\
05\end{array}$ & $\begin{array}{l}4.1 \mathrm{E}- \\
05\end{array}$ & $\begin{array}{l}4.7 \mathrm{E}- \\
05\end{array}$ & $\begin{array}{l}3.8 \mathrm{E}- \\
05\end{array}$ & $\begin{array}{l}3.3 \mathrm{E}- \\
05\end{array}$ & $\begin{array}{l}3.5 \mathrm{E}- \\
05\end{array}$ \\
\hline Dichlorvos & 0.00008 & $0.01 *$ & $0.04^{*}$ & $0.02^{*}$ & $0.01 *$ & $0.01^{*}$ & $0.01 *$ & $0.01 *$ & $0.01 *$ \\
\hline Malaoxon & 0.03 & $5.9 \mathrm{E}-05$ & 0.0002 & $\begin{array}{l}9.4 \mathrm{E}- \\
05\end{array}$ & $\begin{array}{l}5.8 \mathrm{E}- \\
05\end{array}$ & $\begin{array}{l}6.7 \mathrm{E}- \\
05\end{array}$ & $\begin{array}{l}5.4 \mathrm{E}- \\
05\end{array}$ & $\begin{array}{l}4.7 \mathrm{E}- \\
05\end{array}$ & $\begin{array}{l}5.1 \mathrm{E}- \\
05\end{array}$ \\
\hline Methidathion & 0.001 & $3.0 \mathrm{E}-05$ & $8.2 \mathrm{E}-05$ & $\begin{array}{l}4.7 \mathrm{E}- \\
05\end{array}$ & $\begin{array}{l}2.9 \mathrm{E}- \\
05\end{array}$ & $\begin{array}{l}3.3 \mathrm{E}- \\
05\end{array}$ & $\begin{array}{l}2.7 \mathrm{E}- \\
05\end{array}$ & $\begin{array}{l}2.3 \mathrm{E}- \\
05\end{array}$ & $\begin{array}{l}2.5 \mathrm{E}- \\
05\end{array}$ \\
\hline Malathion & 0.03 & 0.0001 & 0.0003 & 0.0002 & 0.0001 & 0.0001 & 0.0001 & $\begin{array}{l}9.4 \mathrm{E}- \\
05\end{array}$ & 0.0001 \\
\hline Methacrifos & 0.006 & 4.1E-05 & 0.0001 & $\begin{array}{l}6.6 \mathrm{E}- \\
05\end{array}$ & $\begin{array}{l}4.1 \mathrm{E}- \\
05\end{array}$ & $\begin{array}{l}4.7 \mathrm{E}- \\
05\end{array}$ & $\begin{array}{l}3.8 \mathrm{E}- \\
05\end{array}$ & $\begin{array}{l}3.3 \mathrm{E}- \\
05\end{array}$ & $\begin{array}{l}3.5 \mathrm{E}- \\
05\end{array}$ \\
\hline Ethoprophos & 0.0004 & $5.9 \mathrm{E}-05$ & 0.0002 & $\begin{array}{l}9.4 \mathrm{E}- \\
05\end{array}$ & $\begin{array}{l}5.8 \mathrm{E}- \\
05\end{array}$ & $\begin{array}{l}6.7 \mathrm{E}- \\
05\end{array}$ & $\begin{array}{l}5.4 \mathrm{E}- \\
05\end{array}$ & $\begin{array}{l}4.7 \mathrm{E}- \\
05\end{array}$ & $\begin{array}{l}5.1 \mathrm{E}- \\
05\end{array}$ \\
\hline Fenamiphos & 0.0008 & $1.2 \mathrm{E}-05$ & 3.3E-05 & $\begin{array}{l}1.9 \mathrm{E}- \\
05\end{array}$ & $\begin{array}{l}1.2 \mathrm{E}- \\
05\end{array}$ & $\begin{array}{l}1.3 \mathrm{E}- \\
05\end{array}$ & $\begin{array}{l}1.1 \mathrm{E}- \\
05\end{array}$ & $\begin{array}{l}9.4 \mathrm{E}- \\
06\end{array}$ & $\begin{array}{l}1.0 \mathrm{E}- \\
05\end{array}$ \\
\hline Quinalphos & 0.001 & 0.0005 & $0.001^{*}$ & 0.0008 & 0.0005 & 0.0005 & 0.0004 & 0.0004 & 0.0004 \\
\hline Coumaphos & 0.001 & $5.3 \mathrm{E}-07$ & $1.5 \mathrm{E}-06$ & $\begin{array}{l}8.4 \mathrm{E}- \\
07\end{array}$ & $\begin{array}{l}5.2 \mathrm{E}- \\
07\end{array}$ & $\begin{array}{l}6.0 \mathrm{E}- \\
07\end{array}$ & $\begin{array}{l}4.9 \mathrm{E}- \\
07\end{array}$ & $\begin{array}{l}4.2 \mathrm{E}- \\
07\end{array}$ & $\begin{array}{l}4.5 \mathrm{E}- \\
07\end{array}$ \\
\hline $\begin{array}{l}\text { Chlorpyriphos- } \\
\text { methyl }\end{array}$ & 0.01 & 0.0002 & 0.0007 & 0.0004 & 0.0002 & 0.0003 & 0.0002 & 0.0002 & 0.0002 \\
\hline Temephos & 0.001 & $3.0 \mathrm{E}-05$ & $8.2 \mathrm{E}-05$ & $\begin{array}{l}4.7 \mathrm{E}- \\
05\end{array}$ & $\begin{array}{l}2.9 \mathrm{E}- \\
05\end{array}$ & $\begin{array}{l}3.3 \mathrm{E}- \\
05\end{array}$ & $\begin{array}{l}2.7 \mathrm{E}- \\
05\end{array}$ & $\begin{array}{l}2.3 \mathrm{E}- \\
05\end{array}$ & $\begin{array}{l}2.5 \mathrm{E}- \\
05\end{array}$ \\
\hline Profenofos & 0.03 & $0.06 *$ & $0.2^{*}$ & $0.1^{*}$ & $0.06 *$ & $0.07 *$ & $0.06 *$ & $0.05^{*}$ & $0.05^{*}$ \\
\hline Pirimiphosmethyl & 0.03 & $1.8 \mathrm{E}-05$ & $4.9 \mathrm{E}-05$ & $\begin{array}{l}2.8 \mathrm{E}- \\
05\end{array}$ & $\begin{array}{l}1.7 \mathrm{E}- \\
05\end{array}$ & $2 \mathrm{E}-05$ & $\begin{array}{l}1.6 \mathrm{E}- \\
05\end{array}$ & $\begin{array}{l}1.4 \mathrm{E}- \\
05\end{array}$ & $\begin{array}{l}1.5 \mathrm{E}- \\
05\end{array}$ \\
\hline Fenitrothion & 0.005 & $0.1^{*}$ & $0.3^{*}$ & $0.2^{*}$ & $0.1 *$ & $0.1 *$ & $0.1 *$ & $0.09 *$ & $0.1^{*}$ \\
\hline Aminocarb & 0.001 & 0.0009 & $0.003^{*}$ & $0.002^{*}$ & 0.0009 & $0.001 *$ & 0.0009 & 0.0007 & 0.0008 \\
\hline Methomyl & 0.0025 & 0.0002 & 0.0005 & 0.0003 & 0.0002 & 0.0002 & 0.0002 & 0.0001 & 0.0001 \\
\hline Aldicarbfragment & 0.001 & 0.0001 & 0.0003 & 0.0002 & 0.0001 & 0.0001 & 0.0001 & $\begin{array}{l}9.4 \mathrm{E}- \\
05\end{array}$ & 0.0001 \\
\hline
\end{tabular}

BDL - Below detection limit, ADI - Acceptable Daily Intake, EDI - Estimated Daily Intake * - EDI greater than ADI (HQ > 1) 


\begin{tabular}{|c|c|c|c|c|c|c|c|c|c|}
\hline \multirow[t]{2}{*}{ Pesticides } & \multirow{2}{*}{$\begin{array}{l}\text { ADI (mg/kg } \\
\text { bw/day) }\end{array}$} & \multicolumn{8}{|c|}{ EDI (mgkg/bw/day) } \\
\hline & & $\begin{array}{l}\text { General } \\
\text { population }\end{array}$ & $<5$ & $5-12$ & $13-19$ & $20-24$ & $25-35$ & $36-49$ & $50+$ \\
\hline Pirimicarb & 0.035 & 0.0001 & 0.0003 & 0.0002 & 0.0001 & 0.0001 & 0.0001 & $\begin{array}{l}9.4 \mathrm{E}- \\
05\end{array}$ & 0.0001 \\
\hline Dioxacarb & 0.001 & $0.02 *$ & $0.06 *$ & $0.03^{*}$ & $0.02^{*}$ & $0.02^{*}$ & $0.02^{*}$ & $0.02^{*}$ & $0.02^{*}$ \\
\hline Carbaryl & 0.0075 & $3.0 \mathrm{E}-05$ & $8.2 \mathrm{E}-05$ & $\begin{array}{l}4.7 \mathrm{E}- \\
05\end{array}$ & $\begin{array}{l}2.9 \mathrm{E}- \\
05\end{array}$ & $\begin{array}{l}3.3 \mathrm{E}- \\
05\end{array}$ & $\begin{array}{l}2.7 \mathrm{E}- \\
05\end{array}$ & $\begin{array}{l}2.3 \mathrm{E}- \\
05\end{array}$ & $\begin{array}{l}2.5 \mathrm{E}- \\
05\end{array}$ \\
\hline Carbofuran & 0.00015 & $5.9 \mathrm{E}-05$ & $0.0002^{*}$ & $\begin{array}{l}9.4 \mathrm{E}- \\
05\end{array}$ & $\begin{array}{l}5.8 \mathrm{E}- \\
05\end{array}$ & $\begin{array}{l}6.7 \mathrm{E}- \\
05\end{array}$ & $\begin{array}{l}5.4 \mathrm{E}- \\
05\end{array}$ & $\begin{array}{l}4.7 \mathrm{E}- \\
05\end{array}$ & $\begin{array}{l}5.1 \mathrm{E}- \\
05\end{array}$ \\
\hline Alanycarb & 0.001 & $0.1^{*}$ & $0.3^{*}$ & $0.2^{*}$ & $0.1^{*}$ & $0.1^{*}$ & $0.1^{*}$ & $0.09 *$ & $0.1^{*}$ \\
\hline Benfuracarb & 0.01 & $0.09 *$ & $0.2^{*}$ & $0.1^{*}$ & $0.09 *$ & $0.1^{*}$ & $0.08 *$ & $0.07 *$ & $0.08 *$ \\
\hline Methiocarb & 0.00025 & 0.0001 & $0.0003^{*}$ & 0.0002 & 0.0001 & 0.0001 & 0.0001 & $\begin{array}{l}9.4 \mathrm{E}- \\
05\end{array}$ & 0.0001 \\
\hline Imidacloprid & 0.06 & 0.003 & 0.008 & 0.005 & 0.003 & 0.003 & 0.003 & 0.002 & 0.003 \\
\hline Acetamiprid & 0.025 & 0.02 & $0.06 *$ & $0.04^{*}$ & 0.02 & $0.03^{*}$ & 0.02 & 0.02 & 0.02 \\
\hline Thiacloprid & 0.01 & $1.2 \mathrm{E}-05$ & 3.3E-05 & $\begin{array}{l}1.9 \mathrm{E}- \\
05\end{array}$ & $\begin{array}{l}1.2 \mathrm{E}- \\
05\end{array}$ & $\begin{array}{l}1.3 \mathrm{E}- \\
05\end{array}$ & $\begin{array}{l}1.1 \mathrm{E}- \\
05\end{array}$ & $\begin{array}{l}9.4 \mathrm{E}- \\
06\end{array}$ & $\begin{array}{l}1.0 \mathrm{E}- \\
05\end{array}$ \\
\hline Bifenthrin & 0.015 & 0.0007 & 0.0015 & 0.001 & 0.0007 & 0.0008 & 0.0007 & 0.0006 & 0.0006 \\
\hline Lambda-Cyhalothrin & 0.0012 & 0.001 & $0.003^{*}$ & $0.002^{*}$ & 0.001 & 0.001 & 0.0009 & 0.0008 & 0.0009 \\
\hline Deltamethrin & 0.01 & $5.9 \mathrm{E}-05$ & 0.0002 & $\begin{array}{l}9.4 \mathrm{E}- \\
05\end{array}$ & $\begin{array}{l}5.8 \mathrm{E}- \\
05\end{array}$ & $\begin{array}{l}6.7 \mathrm{E}- \\
05\end{array}$ & $\begin{array}{l}5.4 \mathrm{E}- \\
05\end{array}$ & $\begin{array}{l}4.7 \mathrm{E}- \\
05\end{array}$ & $\begin{array}{l}5.1 \mathrm{E}- \\
05\end{array}$ \\
\hline Cypermethrin & 0.0016 & $0.003^{*}$ & $0.008 *$ & $0.004^{*}$ & $0.003^{*}$ & $0.003^{*}$ & $0.002^{\star}$ & $0.002^{*}$ & $0.002^{*}$ \\
\hline Carbendazim & 0.02 & 0.0003 & 0.0008 & 0.0005 & 0.0003 & 0.0003 & 0.0003 & 0.0002 & 0.0003 \\
\hline Imazalil & 0.025 & 0.002 & 0.005 & 0.003 & 0.002 & 0.002 & 0.002 & 0.002 & 0.002 \\
\hline Metazachlor & 0.08 & 0.0002 & 0.0005 & 0.0003 & 0.0002 & 0.0002 & 0.0002 & 0.0001 & 0.0002 \\
\hline Metalaxyl & 0.08 & $5.9 \mathrm{E}-05$ & 0.0002 & $\begin{array}{l}9.4 \mathrm{E}- \\
05\end{array}$ & $\begin{array}{l}5.8 \mathrm{E}- \\
05\end{array}$ & $\begin{array}{l}6.7 \mathrm{E}- \\
05\end{array}$ & $\begin{array}{l}5.4 \mathrm{E}- \\
05\end{array}$ & $\begin{array}{l}4.7 \mathrm{E}- \\
05\end{array}$ & $\begin{array}{l}5.1 \mathrm{E}- \\
05\end{array}$ \\
\hline Azaconazole & 0.001 & 0.0002 & 0.0007 & 0.0004 & 0.0002 & 0.0003 & 0.0002 & 0.0002 & 0.0002 \\
\hline Clomazone & 0.133 & $3.5 \mathrm{E}-06$ & $9.9 \mathrm{E}-06$ & $\begin{array}{l}5.6 \mathrm{E}- \\
06\end{array}$ & $\begin{array}{l}3.5 \mathrm{E}- \\
06\end{array}$ & $4 \mathrm{E}-06$ & $\begin{array}{l}3.3 \mathrm{E}- \\
06\end{array}$ & $\begin{array}{l}2.8 \mathrm{E}- \\
06\end{array}$ & $\begin{array}{l}3.0 \mathrm{E}- \\
06\end{array}$ \\
\hline Azoxystrobin & 0.2 & 0.01 & 0.04 & 0.02 & 0.01 & 0.01 & 0.01 & 0.01 & 0.01 \\
\hline Pyrimethanil & 0.17 & 0.0006 & 0.002 & 0.0009 & 0.0006 & 0.0007 & 0.0005 & 0.0005 & 0.0005 \\
\hline Spirotetramat & 0.05 & 0.0002 & 0.0005 & 0.0003 & 0.0002 & 0.0002 & 0.0002 & 0.0001 & 0.0002 \\
\hline Fenhexamid & 0.2 & 0.1 & $0.4^{*}$ & $0.2^{*}$ & 0.1 & 0.2 & 0.1 & 0.1 & 0.1 \\
\hline Fenarimol & 0.01 & 0.002 & 0.006 & 0.003 & 0.002 & 0.002 & 0.002 & 0.002 & 0.002 \\
\hline Fluazifop & 0.004 & $0.007^{*}$ & $0.02^{*}$ & $0.01 *$ & $0.007^{*}$ & $0.008^{*}$ & $0.006^{*}$ & $0.006^{*}$ & $0.006 *$ \\
\hline Flufenoxuron & 0.01 & $1.8 \mathrm{E}-05$ & $4.9 \mathrm{E}-05$ & $\begin{array}{l}2.8 \mathrm{E}- \\
05\end{array}$ & $\begin{array}{l}1.7 \mathrm{E}- \\
05\end{array}$ & $2 \mathrm{E}-05$ & $\begin{array}{l}1.6 \mathrm{E}- \\
05\end{array}$ & $\begin{array}{l}1.4 \mathrm{E}- \\
05\end{array}$ & $\begin{array}{l}1.5 \mathrm{E}- \\
05\end{array}$ \\
\hline Pyriproxyfen & 0.05 & $3.5 \mathrm{E}-06$ & $9.9 \mathrm{E}-06$ & $\begin{array}{l}5.6 \mathrm{E}- \\
06\end{array}$ & $\begin{array}{l}3.5 \mathrm{E}- \\
06\end{array}$ & $4 \mathrm{E}-06$ & $\begin{array}{l}3.3 \mathrm{E}- \\
06\end{array}$ & $\begin{array}{l}2.8 \mathrm{E}- \\
06\end{array}$ & $\begin{array}{l}3.0 \mathrm{E}- \\
06\end{array}$ \\
\hline Quinoxyfen & 0.2 & 0.0002 & 0.0005 & 0.0003 & 0.0002 & 0.0002 & 0.0002 & 0.0001 & 0.0002 \\
\hline Proquinazid & 0.01 & 0.002 & 0.005 & 0.003 & 0.002 & 0.002 & 0.002 & 0.001 & 0.001 \\
\hline
\end{tabular}


Pesticides were detected in all studied fruits and vegetables, with 39 active ingredients (Als) detected in all samples and 18 Als in at least some of the food samples. Fonofos, fenitrothion and fenhexamid concentrations were above the MRLs in watermelon, passion fruit, tomato, cabbage and eggplant. Risk assessment calculations show that EDIs for 18 pesticides were above the ADI in some cases, with HQs that ranged from 1 up to 443 and thus may pose chronic health risks. Children experienced the highest HQs and therefore potentially higher chronic health risks from pesticide residues in fruits and vegetables.

Overall, $29 \%$ of the pesticides we tested for had EDIs over an ADI. This is a high proportion of exceedances compared to other risk assessment studies [46-48]. When calculated by stage along the supply chain and age group, 16 and 18 pesticides respectively had high EDIs are above their ADI. As discussed by JA Vaccaro and FG Huffman [49], age is a key dietary risk factor that should be considered while performing health risk assessment Several fruit and vegetable surveillance studies have estimated EDI and similar EDIs. Studies in Chile, Poland and Kazakhstan had EDIs ranging from $<0.001$ to $5.2[47,50-54]$, which is within the range of our findings.

Many pesticides were detected in all studied fruits and vegetables with levels below the EU MRLs except for Fonofos, fenitrothion and fenhexamid. Our findings are consistent with existing literature showing detection of many pesticides in fruits and vegetables [50, 51, 55, 56]. Like our findings, many past studies have pesticide residue levels that are above MRL values, especially organophosphates like fenitrothion [46, $48,53,54,57-60]$. For example, recent studies in Ghana and Nigeria also found that many pesticides residue levels in produce were above the respective MRLs $[42,61]$. The most frequently detected pesticides that have exceeded MRLs have been organophosphates, carbamates, pyrethroids and neonicotinoids based on studies in Uganda, Ghana, Egypt, Poland and Chile [4, 5, 28, 34, 42, 43, 46, 62], especially in leafy vegetables $[50,51]$. Given that MRLs are determined based on good agricultural practices (GAPs) in field experiments and not necessarily health risks $[63,64]$, consumption of pesticides below the MRLs might exceed health-based exposure benchmarks depending on individual consumption patterns.

Our findings confirm similar findings to other studies carried out in Poland, Nigeria and Saudi Arabia which found that many pesticides had a $\mathrm{HQ}>1[46,65,66]$. On the other hand, literature from Turkey, Poland, Ghana, China and South Korea showed no chronic health risk associated with pesticide residues in fruits and vegetables [53, 54, 58, 60, 67-70]. using probabilistic modelling, Z Eslami, V Mahdavi and B Tajdar-Oranj [57] in Iran found that pesticide residues did not pose health risks to adults and children. When assessed by stage along the supply chain, some pesticide showed a low HQ and consequently lower risk when consumed at farm than at other stages further along the supply chain, such as restaurants and homes. Our findings are similar to those from previous studies which have shown a higher chronic health risk for stages upstream along the chain $[62,71]$. When HQ was assessed by age, children more frequently experienced higher hazard quotients (18 - 13) compared with adults $(11-9)$ with HQS up to 443 , compared with a maximum HQ for adults at XX. Our findings are similar to findings from studies from Chile, Nigeria and China that assessed risk by age which found that chronic health risks were higher in children compared to adults $[50,53,69,72]$.

Our findings have implications on policy and future research. We used the EU MRLs and ADIs to evaluate exposures and risks, these benchmarks are lower and hence more sensitive than other guidelines. For example, Codex Alimentarius guidelines are higher, which would suggest lower health risks based on the exposure we evaluated. There is a need to develop Ugandan standards for MRLs and ADI based on local studies and context. The high HQs demonstrate in our study also demonstrate the need for routine monitoring and surveillance of pesticide residues in foods, especially in fruits and vegetables.

This study has several strengths and limitations. This study is the largest in Uganda to examine pesticide residues in fruits and vegetables; and we interviewed over 2000 residents to obtain information on dietary intake patterns. Dietary consumption data for fruit and vegetable was measured using a contextualised food album and thus presents a true reflection of the study community. We used mean residue concentrations to assess likely average exposures to consumers, but individual variability in eating patterns may result in higher or lower chronic exposures [73]. Additionally, we computed hazard quotients for consumption of individual foods. It is likely that consumers ate several different fruits or vegetables on any given day. In future analyses, we will use probabilistic methods to assess the range of potential exposures and health risks from more realistic diet patterns. We will also apply relative potency factors (RPFs) to assess cumulative health risks for pesticide classes with established RPFS [74]. Fruits and vegetables were not tracked from farm to fork during sampling due cost and time challenges. Future studies examining pesticide residues along the farm to fork chain should track and sample individual produce lots from harvest to the consumer. Additionally, this study was carried out in a primarily urban community and may not represent a typical Ugandan rural setting. Finally, dietary consumption measurement did not cover the broad spectrum of fruits and vegetables but rather focussed on commonly consumed items within the study area (watermelon, passion fruit, tomato, cabbage and eggplant). However, the study area represents a large proportion of the Ugandan population and several commonly eaten foods.

\section{Conclusion}


Sixty-two (62) pesticide residues were detected in fruits and vegetables from farm to fork. Concentrations of fonofos, fenitrothion and fenhexamid were above EU MRLs in watermelon, passion fruit, tomato, cabbages and eggplant. Exposures to sixteen and eighteen pesticides exceeded health-based benchmarks and potentially pose chronic health risks to consumers, especially to children. The study findings demonstrate the urgent need for routine pesticide monitoring and surveillance and risk assessment for fruits and vegetables in local Ugandan markets. There is also need to regulate the levels of pesticide in fruits and vegetables in order to protect consumers, especially the children who present higher chronic health risks.

\section{Abbreviations}

\begin{tabular}{|c|c|}
\hline ADI & Acceptable Daily Intake \\
\hline Als & Active Ingredients \\
\hline BDL & Below Detection Limits \\
\hline BW & Body Weight \\
\hline C & Mean concentration of each Pesticide \\
\hline EDI & Estimated Daily Intake \\
\hline EU MRLs & European Union Maximum Residual Limits \\
\hline FVCR & Fruit and Vegetable Intake Rate \\
\hline GAPs & Good Agricultural Practices \\
\hline $\mathrm{GC}-\mathrm{MS}$ & Gas Chromatography - Mass Spectrometry \\
\hline $\mathrm{HQ}$ & Hazard Quotient \\
\hline KMA & Kampala Metropolitan Area \\
\hline $\mathrm{LC}-\mathrm{MS} / \mathrm{MS}$ & Liquid Chromatography - Tandem Mass Spectrometry \\
\hline LOD & Limit of Detection \\
\hline LOQ & Limit of Quantification \\
\hline MRLs & Maximum Residual Limits \\
\hline NCDs & Noncommunicable Diseases \\
\hline QuEchERS & Quick, Easy, Cheap, Effective, Rugged and Safe \\
\hline RPFs & Relative Potency Factors \\
\hline U.S. FDA & United States Food and Drugs Authority \\
\hline WHO & World Health Organisation \\
\hline
\end{tabular}

\section{Declarations}

\section{Ethics approval and consent to participate}

Ethical approval was granted by the Makerere University School of Public Health Higher Degrees, Research and Ethics Committee (HDREC); and registered by Uganda National Council for Science and Technology (SS 5203). All participants provided written informed consent before their involvement in the study.

\section{Consent for publication}

Not applicable 
The dataset used during the study is available from the corresponding author on reasonable request.

\section{Competing interests}

The authors declare that they have no competing interests.

\section{Funding}

This research was supported by the Consortium for Advanced Research Training in Africa (CARTA). CARTA is jointly led by the African Population and Health Research Center and the University of the Witwatersrand, South Africa and is funded by Sida (Grant No: 54100113 ), Carnegie Corporation of New York (Grant No. G-19-57145), the DELTAS Africa Initiative (Grant No: 107768/Z/15/Z). The DELTAS Africa Initiative is an independent funding scheme of the African Academy of Sciences (AAS)'s Alliance for Accelerating Excellence in Science in Africa (AESA) and supported by the New Partnership for Africa's Development Planning and Coordinating Agency (NEPAD Agency) with funding from the Wellcome Trust (UK) and the UK government. The statements made and views expressed are solely the responsibility of the Authors. Research reported in this publication was partially support by the Fogarty International Center of the National Institutes of Health under Award Number D43TW009340. The content is solely the responsibility of the authors and does not necessarily represent the official views of the National Institutes of Health.

\section{Authors' contribution}

CS: conceived of the study; participated in the design, coordination, and implementation of all study field activities; conducted the statistical analysis; and drafted the manuscript; AB: conceived of the study; participated in the design, and helped to draft the manuscript; JCS: conceived of the study; participated in the design, and helped to draft the manuscript; FS: participated in the design, and helped to draft the manuscript; DG: conceived of the study; participated in the design, and helped to draft the manuscript. All authors read and approved the final manuscript.

\section{Acknowledgement}

The authors wish to thank farmers, market vendors, street vendors, restaurants and homes from whose premises study samples were collected. The authors would also like to thank the pesticide laboratory team at the Government Analytic Laboratory (GAL) especially Evarist Natugonza and Oscar Kibirango for their support during sample collection and analysis as well as Mr. Aggrey Atuhaire from Uganda National Association of Community and Occupational Health for his support with sampling and sample collection. We would also like to thank the study participants and research assistants that took part in the fruit and vegetable intake survey.

\section{References}

1. Aktar MW, Sengupta D, Chowdhury A: Impact of pesticides use in agriculture: their benefits and hazards. Interdisciplinary Toxicology 2009, 2(1):1-12.

2. Matowo NS, Tanner M, Munhenga G, Mapua SA, Finda M, Utzinger J, Ngowi V, Okumu FO: Patterns of pesticide usage in agriculture in rural Tanzania call for integrating agricultural and public health practices in managing insecticide-resistance in malaria vectors. Malaria Journal 2020, 19(1):257.

3. Maggi F, Tang FHM, la Cecilia D, McBratney A: PEST-CHEMGRIDS, global gridded maps of the top 20 crop-specific pesticide application rates from 2015 to 2025. Scientific Data 2019, 6(1):170.

4. Fuhrimann S, Farnham A, Staudacher P, Atuhaire A, Manfioletti T, Niwagaba CB, Namirembe S, Mugweri J, Winkler MS, Portengen L et al: Exposure to multiple pesticides and neurobehavioral outcomes among smallholder farmers in Uganda. Environment internationa/ 2021 , 152:106477.

5. Staudacher P, Fuhrimann S, Farnham A, Mora AM, Atuhaire A, Niwagaba C, Stamm C, Eggen RI, Winkler MS: Comparative Analysis of Pesticide Use Determinants Among Smallholder Farmers From Costa Rica and Uganda. Environmental health insights 2020, 14:1178630220972417.

6. Hill MP, Macfadyen S, Nash MA: Broad spectrum pesticide application alters natural enemy communities and may facilitate secondary pest outbreaks. PeerJ 2017, 5:e4179-e4179.

7. Ministry of Agriculture Animal Industry and Fisheries: Register of agricultural chemical registered under section 4 of the agricultural chemicals (control) act, 2006 at 6th April 2018. In: Register of agricultural chemical registered under section 4 of the agricultural chemicals (control) act, 2006. Edited by Fisheries MoAAla. Entebbe: Ministry of Agriculture Animal Industry and Fisheries; 2018.

8. Ngabirano H, Birungi G: Pesticide use in vegetable production in rural Uganda -A case study of Kabale District, South western Uganda. African Journal of Agricultural Research 2020, 16(11):1573-1581.

Page $14 / 19$ 
9. Akkad R, Schwack W: Multi-enzyme inhibition assay for the detection of insecticidal organophosphates and carbamates by highperformance thin-layer chromatography applied to determine enzyme inhibition factors and residues in juice and water samples. Journal of chromatography B, Analytical technologies in the biomedical and life sciences 2010, 878(17-18):1337-1345.

10. Vale A, Lotti M: Organophosphorus and carbamate insecticide poisoning. Handbook of clinical neurology 2015, 131:149-168.

11. Sagiv SK, Bruno JL, Baker JM, Palzes V, Kogut K, Rauch S, Gunier R, Mora AM, Reiss AL, Eskenazi B: Prenatal exposure to organophosphate pesticides and functional neuroimaging in adolescents living in proximity to pesticide application. Proceedings of the National Academy of Sciences 2019, 116(37):18347-18356.

12. Laskowski D.A.: Physical and Chemical Properties of Pyrethroids. In: Reviews of environmental contamination and toxicology. Volume 174, edn. Edited by G.W. W. New York, NY: Springer; 2002.

13. Bonmatin JM, Giorio C, Girolami V, Goulson D, Kreutzweiser DP, Krupke C, Liess M, Long E, Marzaro M, Mitchell EAD et al: Environmental fate and exposure; neonicotinoids and fipronil. Environmental science and pollution research international 2015, 22(1):35-67.

14. Soderlund DM: Chapter 77 - Toxicology and Mode of Action of Pyrethroid Insecticides. In: Hayes' Handbook of Pesticide Toxicology (Third Edition). edn. Edited by Krieger R. New York: Academic Press; 2010: 1665-1686.

15. Simaremare SRS, Hung C-C, Hsieh C-J, Yiin L-M: Relationship between Organophosphate and Pyrethroid Insecticides in Blood and Their Metabolites in Urine: A Pilot Study. International journal of environmental research and public health 2019, 17(1):34

16. Bradberry SM, Cage SA, Proudfoot AT, Vale JA: Poisoning due to pyrethroids. Toxicological reviews 2005, 24(2):93-106.

17. Pitzer EM, Williams MT, Vorhees CV: Effects of pyrethroids on brain development and behavior: Deltamethrin. Neurotoxicology and Teratology 2021, 87:106983.

18. Vester Al, Chen M, Marsit CJ, Caudle WM: A Neurodevelopmental Model of Combined Pyrethroid and Chronic Stress Exposure. Toxics 2019, $7(2): 24$

19. Taillebois E, Cartereau A, Jones AK, Thany SH: Neonicotinoid insecticides mode of action on insect nicotinic acetylcholine receptors using binding studies. Pesticide Biochemistry and Physiology 2018, 151:59-66.

20. Cartereau A, Taillebois E, Le Questel JY, Thany SH: Mode of Action of Neonicotinoid Insecticides Imidacloprid and Thiacloprid to the Cockroach Pamea7 Nicotinic Acetylcholine Receptor. Int J Mol Sci 2021, 22(18).

21. Houchat J-N, Cartereau A, Le Mauff A, Taillebois E, Thany SH: An Overview on the Effect of Neonicotinoid Insecticides on Mammalian Cholinergic Functions through the Activation of Neuronal Nicotinic Acetylcholine Receptors. Int J Environ Res Public Health 2020, 17(9):3222.

22. Cimino AM, Boyles AL, Thayer KA, Perry MJ: Effects of Neonicotinoid Pesticide Exposure on Human Health: A Systematic Review Environmental health perspectives 2017, 125(2):155-162.

23. PAN: Hazardous pesticide and health impacts in Africa. In: Food and fairness briefying No 6. vol. 6. United Kingdom: PAN UK; 2018.

24. Nicolopoulou-Stamati P, Maipas S, Kotampasi C, Stamatis P, Hens L: Chemical Pesticides and Human Health: The Urgent Need for a New Concept in Agriculture. Frontiers in Public Health 2016, 4:148.

25. Coker E, Chevrier J, Rauch S, Bradman A, Obida M, Crause M, Bornman R, Eskenazi B: Association between prenatal exposure to multiple insecticides and child body weight and body composition in the VHEMBE South African birth cohort. Environment international 2018 , 113:122-132.

26. Chiu YH, Williams PL, Gillman MW, Gaskins AJ, Minguez-Alarcon L, Souter I, Toth TL, Ford JB, Hauser R, Chavarro JE: Association Between Pesticide Residue Intake From Consumption of Fruits and Vegetables and Pregnancy Outcomes Among Women Undergoing Infertility Treatment With Assisted Reproductive Technology. JAMA internal medicine 2018, 178(1):17-26.

27. FOA: FAOSTAT: The State of Food Security Indicators (Food Security and Nutrition). In., August 20, 2021 edn. Rome: Food and Agricultural Organisation; 2021

28. Kaye E, Nyombi A, Mutambuze IL, Muwesa R: Mancozeb Residue on Tomatoes in Central Uganda. Journal of Health and Pollution 2015, 5(8):1-6.

29. Grewel AS, Singla A, Kamboj P, Dua JS: Pesticide Residues in Food Grains, Vegetables and Fruits: A Hazard to Human Health. Journal of Medicinal Chemistry and Toxicology 2017, 2(1):40-46.

30. World Health Organisation: Global Action Plan for the Prevention and Control of NCDs 2013-2020. In. Geneva: Switzerland World Health Organisation; 2013: 103.

31. Ssemugabo C, Ssempebwa J, Bradman A, Sille F, Guwatudde D: Fruits and vegetables consumption in Kampala Metropolitan Area, Uganda: a household survey In. Edited by University M. Kampala: Uganda; 2021: 16.

32. Kabwama SN, Bahendeka SK, Wesonga R, Mutungi G, Guwatudde DJAoPH: Low consumption of fruits and vegetables among adults in Uganda: findings from a countrywide cross-sectional survey. Archives of Public Health 2019, 77(1):4. 
33. Ssemugabo C, Bradman A, Ssempebwa JC, Sillé F, Guwatudde D: Pesticide residues in fresh fruit and vegetables from farm to fork in the Kampala Metropolitan Area, Uganda In: Food Additives and Contaminants Part B. Edited by University M; 2021.

34. Atuhaire A, Kaye E, Mutambuze IL, Matthews G, Friedrich T, Jørs E: Assessment of Dithiocarbamate Residues on Tomatoes Conventionally Grown in Uganda and the Effect of Simple Washing to Reduce Exposure Risk to Consumers. Environmental health insights 2017,

11:1178630217712218.

35. UBOS: Uganda Bureau of Statistics's Statistical Abstract 2018. In. Kampala: Uganda Uganda Bureau of Statistics 2018.

36. Kasimbazi E: Urban Expansion in the Greater Kampala Metropolitan Area, Uganda. In. Nairobi: UN Habitat 2016.

37. El-Zaher TR, Nasr IN, Mahmoud HA: Behavior of Some Pesticide residues in and on Tomato and Kidney Beans Fruits Grown in Open Field. American-Eurasian Journal of Toxicological Sciences 2011, 3(3):213-218.

38. Food and Agriculture Organisation: Recommended methods of sampling for pesticide residues for the determination of compliance with MRLs. Rome: Food and agriculture Organisation of United Nations; 1999.

39. Anastassiades M, Lehotay S, Štajnbaher D: Quick, easy, cheap, effective, rugged, and safe (QuEChERS) approach for the determination of pesticide residues; 2002.

40. WHO: The WHO STEPwise approach to noncommunicable disease risk factor surveillance (STEPS). In. Geneva: World Health organisation; 2017.

41. Pesticide residues [https://ec.europa.eu/food/plant/pesticides/eu-pesticides-database/mrls/?event=search.pr]

42. Fosu PO, Donkor A, Ziwu C, Dubey B, Kingsford-Adaboh R, Asante I, Nyarko S, Tawiah R, Nazzah N: Surveillance of pesticide residues in fruits and vegetables from Accra Metropolis markets, Ghana, 2010-2012: a case study in Sub-Saharan Africa. Environmental Science Pollution Research 2017, 24(20):17187-17205.

43. Issa AB, Yassin K, Loutfy N, Ahmed MT: Risk assessment of some organic contaminants: a case study based on food consumption in Tanta and Ismailia cities, Egypt. Environmental science and pollution research international 2018, 25(34):34212-34220.

44. Gad Alla SA, Loutfy NM, Shendy AH, Ahmed MT: Hazard index, a tool for a long term risk assessment of pesticide residues in some commodities, a pilot study. Regulatory Toxicology and Pharmacology 2015, 73(3):985-991.

45. Chen C, Qian Y, Chen Q, Tao C, Li C, Li Y: Evaluation of pesticide residues in fruits and vegetables from Xiamen, China. Food Control 2011, 22(7):1114-1120.

46. Szpyrka E, Kurdziel A, Słowik-Borowiec M, Grzegorzak M, Matyaszek A: Consumer exposure to pesticide residues in apples from the region of south-eastern Poland. Environ Monit Assess 2013, 185(11):8873-8878.

47. Lozowicka B, Abzeitova E, Sagitov A, Kaczynski P, Toleubayev K, Li A: Studies of pesticide residues in tomatoes and cucumbers from Kazakhstan and the associated health risks. Environ Monit Assess 2015, 187(10):609.

48. Mebdoua S, Lazali M, Ounane SM, Tellah S, Nabi F, Ounane G: Evaluation of pesticide residues in fruits and vegetables from Algeria. Food additives \& contaminants Part B, Surveillance 2017, 10(2):91-98.

49. Vaccaro JA, Huffman FG: Dietary Risk Factors by Race/Ethnicity, Age-Group, and Gender in a Representative Sample of US Older Adults. The journal of nutrition, health \& aging 2017, 21(6):704-709.

50. Elgueta S, Valenzuela M, Fuentes M, Meza P, Manzur JP, Liu S, Zhao G, Correa A: Pesticide Residues and Health Risk Assessment in Tomatoes and Lettuces from Farms of Metropolitan Region Chile. Molecules (Basel, Switzerland) 2020, 25(2).

51. Elgueta S, Fuentes M, Valenzuela M, Zhao G, Liu S, Lu H, Correa A: Pesticide residues in ready-to-eat leafy vegetables from markets of Santiago, Chile, and consumer's risk. Food Additives \& Contaminants: Part B 2019, 12(4):259-267.

52. Elgueta S, Moyano S, Sepúlveda P, Quiroz C, Correa A: Pesticide residues in leafy vegetables and human health risk assessment in North Central agricultural areas of Chile. Food additives \& contaminants Part B, Surveillance 2017, 10(2):105-112.

53. Si WS, Wang SY, Zhang YD, Kong C, Bai B: Pesticides and risk assessment in Shanghai fruit and raw eaten vegetables. Food additives \& contaminants Part B, Surveillance 2021:1-11.

54. Szpyrka E, Słowik-Borowiec M: Consumer health risk to pesticide residues in Salvia officinalis L. and its infusions. Journal of environmental science and health Part B, Pesticides, food contaminants, and agricultural wastes 2019, 54(1):14-19.

55. Jallow MFA, Awadh DG, Albaho MS, Devi VY, Ahmad N: Monitoring of Pesticide Residues in Commonly Used Fruits and Vegetables in Kuwait. International Journal of Environmental Research and Public Health 2017, 14(8):833.

56. López-Dávila E, Houbraken M, De Rop J, Claus G, Wumbei A, Romero Romero O, Spanoghe P: Pesticide traces in local crops of Sancti Spíritus, Cuba: risk assessment study. International Journal of Food Contamination 2021, 8(1):1.

57. Eslami Z, Mahdavi V, Tajdar-Oranj B: Probabilistic health risk assessment based on Monte Carlo simulation for pesticide residues in date fruits of Iran. Environmental science and pollution research international 2021, 28(31):42037-42050. 
58. Kazar Soydan D, Turgut N, Yalçın M, Turgut C, Karakuş PBK: Evaluation of pesticide residues in fruits and vegetables from the Aegean region of Turkey and assessment of risk to consumers. Environmental science and pollution research international 2021, 28(22):2751127519.

59. Toptanci İ, Kiralan M, Ramadan MF: Levels of pesticide residues in fruits and vegetables in the Turkish domestic markets. Environmental science and pollution research international 2021, 28(29):39451-39457.

60. Akoto O, Gavor S, Appah MK, Apau J: Estimation of human health risk associated with the consumption of pesticide-contaminated vegetables from Kumasi, Ghana. Environ Monit Assess 2015, 187(5):244.

61. Adeleye AO, Sosan MB, Oyekunle JAO: Dietary exposure assessment of organochlorine pesticides in two commonly grown leafy vegetables in South-western Nigeria. Heliyon 2019, 5(6):e01895.

62. Akomea-Frempong S, Ofosu IW, Owusu-Ansah Ed-GJ, Darko G: Health risks due to consumption of pesticides in ready-to-eat vegetables (salads) in Kumasi, Ghana. International Journal of Food Contamination 2017, 4(1):13.

63. Fothergill A, Abdelghani AJWToE, Environment t: A review of pesticide residue levels and their related health exposure risks. 2013, 170:195205.

64. Salazar JDJOoPM: The OECD MRL Calculator. 2011, 22(2):77-79.

65. Odewale GO, Sosan MB, Oyekunle JAO, Adeleye AO: Human health risk assessment of dichlorodiphenyltrichloroethane (DDT) and hexachlorocyclohexane $(\mathrm{HCH})$ pesticide residues in fruits and vegetables in Nigeria. Environmental science and pollution research international 2021.

66. Picó Y, El-Sheikh MA, Alfarhan AH, Barceló D: Target vs non-target analysis to determine pesticide residues in fruits from Saudi Arabia and influence in potential risk associated with exposure. Food and chemical toxicology : an international journal published for the British Industrial Biological Research Association 2018, 111:53-63.

67. Szpyrka E: Assessment of consumer exposure related to improper use of pesticides in the region of southeastern Poland. Environ Monit Assess 2015, 187(1):4140.

68. Park DW, Yang YS, Lee YU, Han SJ, Kim HJ, Kim SH, Kim JP, Cho SJ, Lee D, Song N et al: Pesticide Residues and Risk Assessment from Monitoring Programs in the Largest Production Area of Leafy Vegetables in South Korea: A 15-Year Study. Foods 2021, 10(2).

69. Zhang Y, Si W, Chen L, Shen G, Bai B, Zhou C: Determination and dietary risk assessment of 284 pesticide residues in local fruit cultivars in Shanghai, China. Sci Rep 2021, 11(1):9681.

70. Yi YJ, Joung HJ, Kum JY, Hwang IS, Kim MS: Pesticide residues in vegetables and risk assessment for consumers in Korea during 20102014. Food additives \& contaminants Part A, Chemistry, analysis, control, exposure \& risk assessment 2020, 37(8):1300-1313.

71. Jacxsens L, Stals A, De Keuckelaere A, Deliens B, Rajkovic A, Uyttendaele M: Quantitative farm-to-fork human norovirus exposure assessment of individually quick frozen raspberries and raspberry puree. International journal of food microbiology 2017, $242: 87-97$.

72. Adeleye AO, Sosan MB, Oyekunle JAO: Occurrence and Human Health Risk of Dichlorodiphenyltrichloroethane (DDT) and Hexachlorocyclohexane (HCH) Pesticide Residues in Commonly Consumed Vegetables in Southwestern Nigeria. J Health Pollut 2019, 9(23):190909.

73. Szpyrka E, Kurdziel A, Matyaszek A, Podbielska M, Rupar J, Słowik-Borowiec M: Evaluation of pesticide residues in fruits and vegetables from the region of south-eastern Poland. Food Control 2015, 48:137-142.

74. U.S. Environmental Protection Agency: Guidance on Cumulative Risk Assessment of Pesticide Chemicals That Have a Common Mechanism of Toxicity. In. Washington: Office of Pesticide Programs U.S. Environmental Protection Agency $2002: 90$.

\section{Figures}




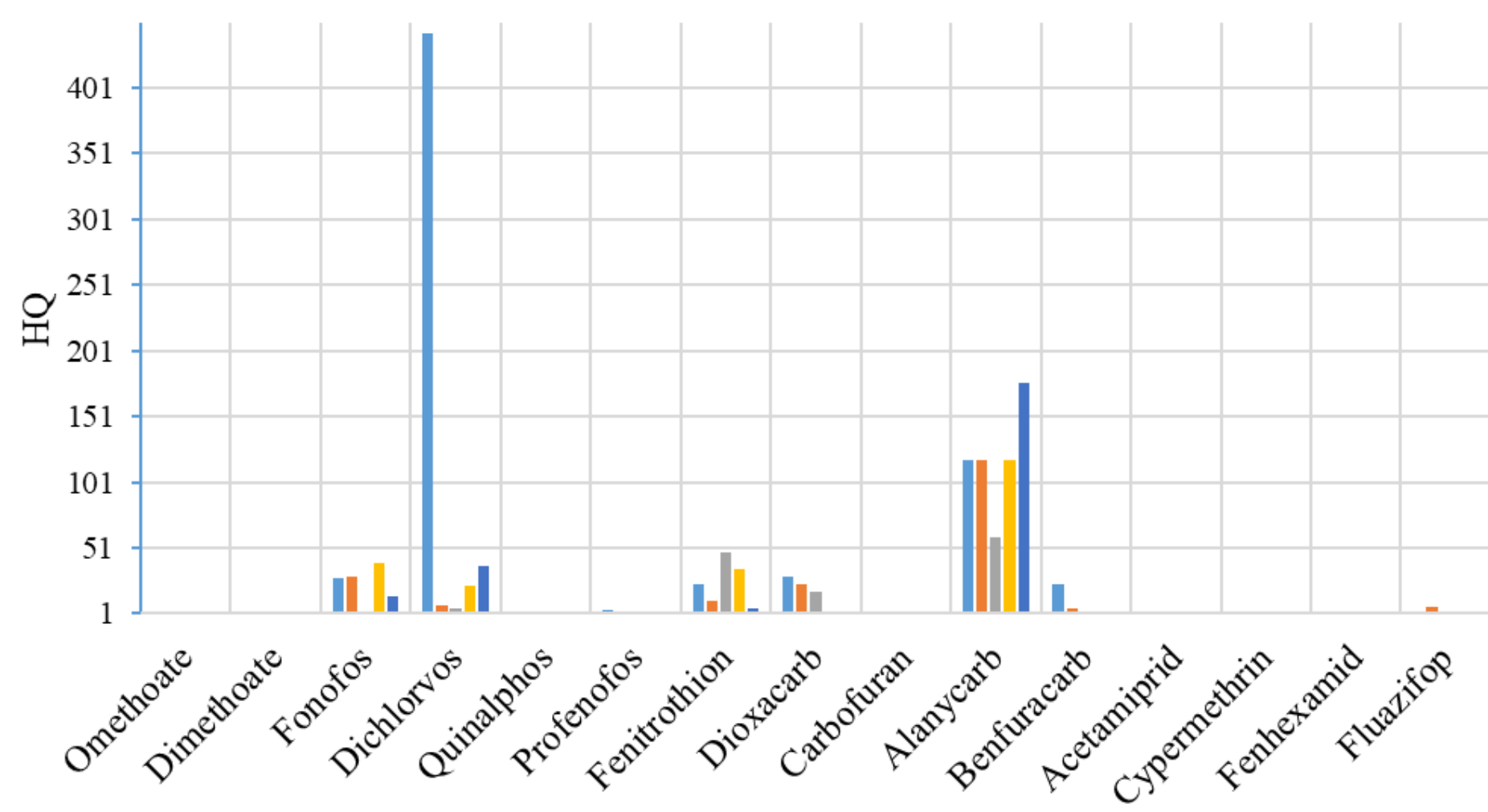

- Farm $\square$ Market $\backsim$ Street $\llbracket$ Restaurant $\backsim$ Home

Figure 1

Hazard quotients for various pesticide residuals, for fruits and vegetables by stage of consumption along the chain

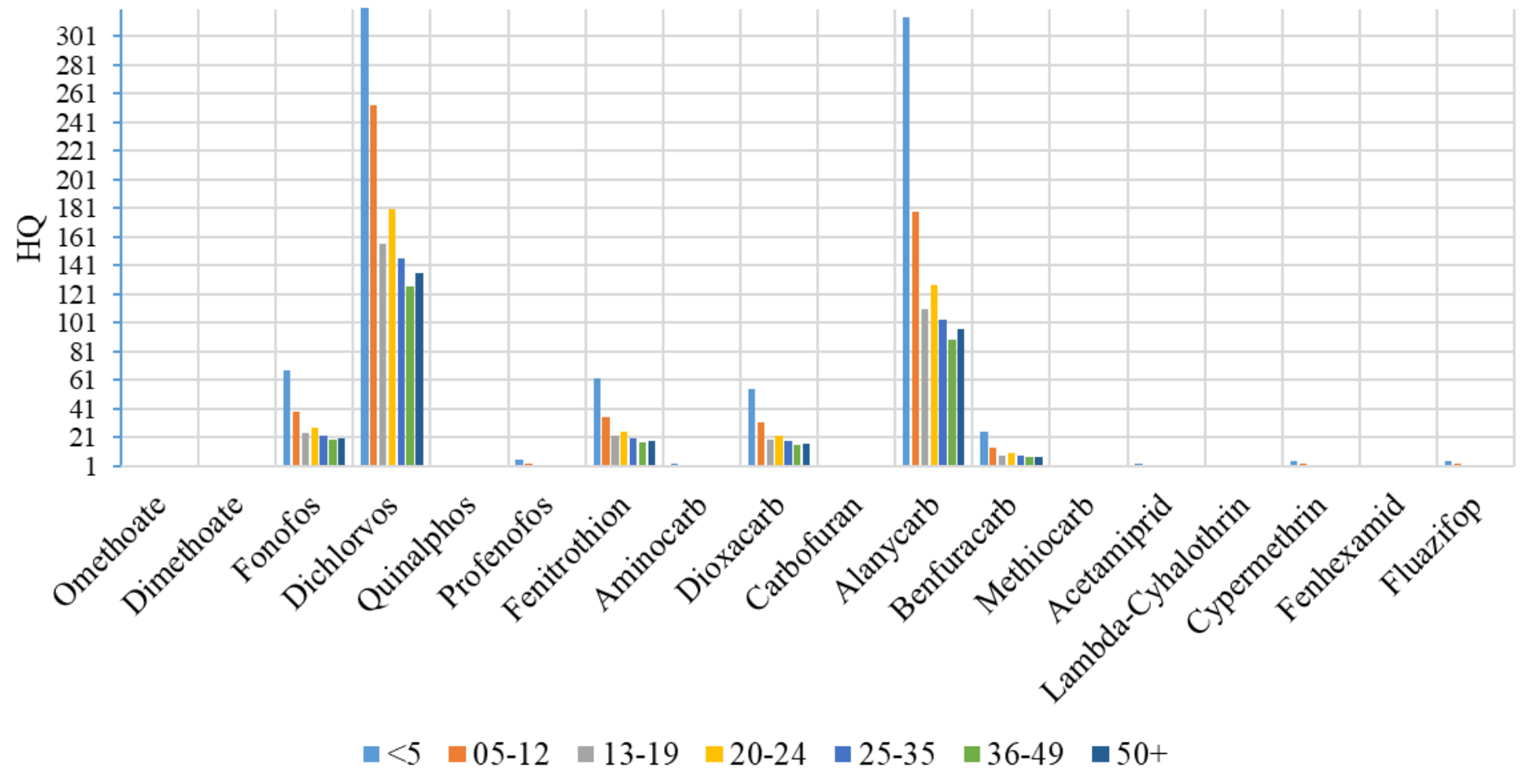

Figure 2 
Hazard quotients for various pesticide residuals, for fruits and vegetables by age group

\section{Supplementary Files}

This is a list of supplementary files associated with this preprint. Click to download.

- SupllementaryMaterials.docx 